\title{
Uniform Dynamic Self-Stabilizing Leader Election
}

\author{
Shlomi Dolev, Amos Israeli, and Shlomo Moran
}

\begin{abstract}
A distributed system is self-stabilizing if it can be started in any possible global state. Once started the system regains its consistency by itself, without any kind of outside intervention. The self-stabilization property makes the system tolerant to faults in which processors exhibit a faulty behavior for a while and then recover spontaneously in an arbitrary state. When the intermediate period in between one recovery and the next faulty period is long enough, the system stabilizes. A distributed system is uniform if all processors with the same number of neighbors are identical. A distributed system is dynamic if it can tolerate addition or deletion of processors and links without reinitialization. In this work, we study uniform dynamic self-stabilizing protocols for leader election under readwrite atomicity. Our protocols use randomization to break symmetry. The leader election protocol stabilizes in $O(\Delta \mathcal{D} \log n)$ time when the number of the processors is unknown and $O(\Delta \mathcal{D})$, otherwise. Here $\Delta$ denotes the maximal degree of a node, $\mathcal{D}$ denotes the diameter of the graph and $n$ denotes the number of processors in the graph. We introduce self-stabilizing protocols for synchronization that are used as building blocks by the leader-election algorithm. We conclude this work by presenting a simple, uniform, self-stabilizing ranking protocol.
\end{abstract}

Index Terms-Self-stabilizing systems, leader election, distributed algorithms, randomized distributed algorithms, synchronization.

\section{INTRODUCTION}

L EADER-ELECTION is one of the fundamental tasks in distributed computing. Roughly speaking, a protocol that solves this task requires that when its execution terminates, a single processor is designated as a leader and every processor knows whether it is a leader or not. By definition, whenever a leader-election protocol terminates successfully, the system is in a nonsymmetric global state. In many cases, once a leader is elected the distributed task is solved by means of a central solution i.e., the leader controls the activity in the distributed system. A partial list of distributed tasks that can be easily realized in the presence of a leader include: consensus, resource allocation and synchronization. Therefore, it is not surprising that the leader election problem has been extensively studied (see for example, [18], [20], [23], [24], [25], [30]).

The number of processors and the sometimes noisy communication media in a distributed system impose the need for a fault tolerant design. One strong notion of fault tolerance is self-stabilization. Roughly speaking, a selfstabilizing protocol can cope with any kind of faults in the history. A distributed system is self-stabilizing if it can be started in any possible global state. Once started, the system runs for a while until it reaches a legitimate global state in which the system is consistent. The self-stabilization property makes the system tolerant to faults in which processors exhibit a faulty behavior for a while and then recover

- S. Dolev is with the Department of Mathematics and Computer Science, Ben-Gurion University, Beer-Sheva, 84105, Israel.

E-mail:dolev@cs.bgu.ac.il.

- A. Israeli is with Intel, Haifa, 31015 Israel.

- S. Moran is with the Computer Science Department, Israel Institute of Technology, Technion, Israel.

Manuscript received May 28, 1994

For information on obtaining reprints of this article, please send e-mail to: transpds@computer.org, and reference IEEECS Log Number D95228. spontaneously in an arbitrary state. When the intermediate period between one recovery and the next faulty period is long enough, the system stabilizes.

Any leader-election protocol that has a symmetric initial state requires some means of symmetry breaking. In idbased systems each processor has a unique identifier called the processor's $i d$, hence the system has no symmetric global-state. A semiuniform system has two kinds of processors: a unique predetermined processor of one type and all other processors are of the other type. The unique processor serves as a leader and prevents the existence of symmetric configurations. In uniform ${ }^{1}$ leader-election protocols, all processors are identical, the initial state is symmetric and symmetry is broken by randomization. Such setting is very useful when the processors are fabricated in a uniform process without assigning each processor by a unique identifier. Note that even in the semiuniform setting some outside coordination is required to ensure that there exists a unique processor in the system. This coordination is specially hard in dynamic environment where processor may join and leave the system during the execution. Another motivation for the uniform system setting is the possibility of outside coordination mistakes such as assigning the same identifier to two processors. A uniform system does not rely on such outside coordination.

\subsection{Previous Work}

Self-stabilizing systems were introduced in the seminal paper of Dijkstra, [14]. In that paper, Dijkstra presents three semiuniform, self-stabilizing, ring protocols for mutualexclusion. Other semiuniform, mutual-exclusion, selfstabilizing ring protocols which work under a stronger adversary, called the distributed demon were presented by Brown, Gouda, and Wu in [10] and by Burns in [12]. Two

1. Uniform systems are also referred to as anonymous. 
papers considered self-stabilizing, mutual-exclusion protocols for general (connected) graphs: The first was authored by Tchuente, in [31], who presented a nonuniform protocol for that problem. A semiuniform protocol for the same problem was presented by Dolev, Israeli, and Moran in [15]. The work of [15] was the first to propose the readwrite atomicity model and their protocol is the first protocol that is self-stabilizing under this model. A selfstabilizing, id-based protocol for mutual exclusion in complete graphs is presented by Lamport, in [27]. This protocol has exponential space complexity. Protocols for leader election in the $i d$-based model for a general graph are presented by Arora and Gouda, in [5] and by Afek, Kutten, and Yung in [6]. Both protocols assume read-write atomicity.

So far, there are very few uniform self-stabilizing protocols: Burns and Pachl present a uniform, deterministic, selfstabilizing, mutual exclusion protocol for rings of prime size in [11]. Randomized, uniform, self-stabilizing protocols for mutual exclusion in a general graph and for ring orientation are presented by Israeli and Jalfon in [21] and [22] respectively. If one could run id-based and semiuniform protocols on a uniform system, the repertoire of uniform self-stabilizing protocols would be considerably enlarged. Let $\mathcal{P} \mathcal{R}$ be an arbitrary semiuniform, self-stabilizing protocol. To run $\mathcal{P} \mathcal{R}$ on a uniform system we employ a uniform, self-stabilizing leader-election protocol and combine it with $\mathcal{P R}$, using fair protocol composition-a technique presented in [15]. Our self-stabilizing ranking protocol assigns the processors with unique identifiers. Similarly to a semiuniform self-stabilizing protocol, it is possible to combine an id based protocol $\mathcal{P} \mathcal{R}$ with the ranking protocol. This combined protocol can be applied to uniform systems.

Our protocol is the only protocol which solves the problem without any prior knowledge on the communication graph. Assuming some known bound on the graph's diameter solutions to the same problem have been suggested independently in [6], [4] and in [8]. The time complexity of all these solutions is inferior to the time complexity of our protocol.

There are many non-self-stabilizing distributed protocols for leader election. We now survey the most related ones: Deterministic, leader-election protocols in id-based systems are presented in [18], [20], [25], [24]. Uniform, randomized, leader-election protocols are presented in [23], [30], [28]. Other protocols for uniform systems appear in [2], [1].

\subsection{The Current Work}

In [17] we presented the computation model, a proof technique for randomized algorithms and two uniform selfstabilizing leader election protocols for complete communication graphs. In the current work we present a uniform, dynamic, self-stabilizing, leader election protocol for general graph systems.

The resources used for stabilization are execution time and memory. The following complexity measures capture the amount of resources required by our protocols:

- Stabilization time and

- Space.

The stabilization time of a self-stabilizing protocol is the maximal time (measured in asynchronous rounds which is precisely defined in the next section) it takes the system to reach a legitimate configuration where the maximum is taken over all possible executions. We consider stabilization time a very important complexity measure and carefully analyze our protocols' stabilization time. To do that, we use the sl-game method [17], for proving upper bounds on the time complexity of randomized distributed protocols. During the execution of our protocol each processor may extend the amount of memory it uses. The space complexity of our protocol is the expected number of extension bits per a processor. According to the above definition, the space complexity of our protocol is $O(\log n)$.

The rest of this paper is organized as follows: In Section 2, we present the formal model and requirements for uniform, self-stabilizing protocols. Section 3 presents the general graph leader election protocol, and the selfstabilizing synchronization protocols. Section 4 presents the self-stabilizing ranking protocol. Conclusions are in Section 5. The Appendix contains the notations and terms.

\section{Model and Requirements}

The model is identical to the one presented in [17]. A uniform distributed system consists of $n$ processors denoted by $P_{1}, P_{2}, \ldots, P_{n}$. Processors are anonymous, they do not have identities. The subscript $1,2, \ldots, n$ are used for ease of notation only. Each processor communicates with all other processors using a single writer, multireader register which is serializable with respect to read and write actions. For the sake of clarity, we assume that every processor knows the exact contents of the register that it is writing to. ${ }^{2}$

For ease of presentation, we regard each processor as a $C P U$ whose program is composed of atomic steps. An atomic step of a processor consists of an internal computation followed by a terminating action. The terminating actions are read, write and coin toss. We assume that the state of a processor fully describes its internal state and the value written in its register. Denote the set of states of $P_{i}$ by $S_{i}$. A configuration, $c \in\left(S_{1} \times S_{2} \times \ldots S_{n}\right)$, of the system is a vector of states of all processors.

Processor activity is managed by a scheduler. In any given configuration, the scheduler activates a single processor which executes a single atomic step. To ensure correctness of the protocols, we regard the scheduler as an adversary. The scheduler is assumed to have unlimited resources, and it chooses the next activated processor on line, using the full information on the execution so far. An execution of the system is a finite or an infinite sequence of configurations $E=\left(c_{1}, c_{2}, \ldots\right)$ such that for $i=1,2, \ldots, c_{i+1}$ is reached from $c_{i}$ by a single atomic step of some processor. A fair execution is an infinite execution in which every processor executes atomic steps infinitely often. A scheduler $S$ is fair if, for any configuration $c$, with probability one, an execution starting from $c$ in which processors are activated by $S$ is fair.

In a distributed asynchronous system, each processor may operate at any nonconstant rate and different proces-

2. One may assume that every processor refreshes the contents of its register periodically. 
sors might be slow in different parts of the execution. The following definition of round complexity attempts to give a complexity measure in which the unfair behavior of the adversary is neutralized, by capturing the rate of action of the slowest processor in any segment of the execution. Given an execution $E$, we define the first round of $E$ to be the minimal prefix of $E, E^{\prime}$, containing atomic steps of every processor in the system. Let $E^{\prime \prime}$ be the suffix of $E$ for which $E=E^{\prime} \circ E^{\prime \prime}$. The second round of $E$ is the first round of $E^{\prime \prime}$, and so on. For any given execution, $E$, the round complexity (which is sometimes called the execution time) of $E$ is the number of rounds in $E$. Under this definition the time to complete a single round is unbounded and depends on the fairness of the adversary. Any self-stabilizing application that uses our protocol as a subroutine would probably also require fair behavior to stabilize and its complexity will be proportional to the stabilization complexity of our protocol.

We proceed by defining the self-stabilization requirements for randomized distributed systems. A behavior of a system is specified by a set of executions. Define a task $L E$ to be a set of executions which are called legitimate executions. A configuration $c$ is safe with respect to a task $L E$ and a protocol $\mathcal{P R}$ if any fair execution of $\mathcal{P R}$ starting from $c$ belongs to $L E$. Finally, a protocol $\mathcal{P R}$ is randomized selfstabilizing for a task $L E$, if starting with any system configuration and considering any fair scheduler, the protocol reaches a safe configuration within an expected number of rounds which is bounded by some constant $C$ (the constant $C$ may depend on $n$, the number of processors in the system).

\section{Leader Election in General Graphs}

\subsection{Informal Description of the Protocol}

In this protocol each system configuration $c$ encodes a directed graph called the FSG (father-son relation graph) of $c$ and denoted by $F S G(c)$. A safe configuration in this protocol is a configuration whose FSG is a single in-tree (called tree in the sequel) which contains all processors and for which during any execution that begins in that configuration the tree is not changed; the root of the FSG is the elected leader.

The protocol consists of two conceptual phases which are called cycle elimination and tree fusion. During the cycle elimination phase all cycles in the FSG are removed. In the tree fusion phase the number of trees in the FSG is reduced until it consists of a single tree. Coin tosses are used in the tree fusion phase in order to break symmetry between trees. Normal operation and completion of tree fusion depends crucially on completion of the cycle elimination phase. By the nature of self-stabilizing protocols the completion of the cycle elimination phase is undetectable locally by the processors. Hence, the cycle elimination phase does not terminate and is executed together with tree fusion phase.

The FSG is defined by a relation between neighbors called father-son relation. Each processor can either be a root or can have a father, which is one of its neighbors. If $P_{i}$ is the father of $P_{j}$ in configuration $c$ then there is a directed edge from $P_{j}$ to $P_{i}$ in FSG $(c)$. Thus, in any configuration $c$, there are at most $n$ edges in $F S G(c)$. Each tree of $F S G(c)$ is identified by a binary string which is called tree-identifier, and abbreviated tid. A root is the only processor which changes the tree's tid; this is always done by extending the tid with a randomly chosen bit. Each non-root processor repeatedly copies its father's tid. Hence in every execution, eventually all processors in a tree $T$, hold a prefix of the tid of the root of $T$.

To achieve cycle elimination each processor computes the distance to the root of its tree. Every processor computes the distance to the root of its tree by adding one to the distance of its father from the root. Whenever the processor realizes that this distance "grows" it (assumes that it is part of a cycle in FSG and) cuts the edge to its father and becomes a separate root. After this phase is completed FSG is a forest of trees.

To reduce the number of trees to one we first ensure that eventually there is a unique tid in the system. Each processor repeatedly scans its neighbors tids. Whenever a processor $P_{i}$ discovers a neighbor $P_{j}$ whose tid is larger than its own tid, $P_{i}$ takes $P_{j}$ to be its father. If previously $P_{i}$ is a root, the number of trees is reduced by one. We prove that taking a new father never introduces new cycles in FSG(c). This however does not ensure that eventually there is a single tree since there might be several trees with the same tid.

A root processor discovers that there are other roots with the (same) maximal tid by repeatedly recoloring its tree using a global synchronization protocol. Each recoloring starts from the root which chooses the new color randomly. The root waits for each of its sons to confirm that every node in its subtree is recolored. Once the entire tree is recolored the root chooses a new color once more, and so on. A processor of a tree $T$ detects the existence of another tree $T^{\prime}$ with the same tid, by observing that one of its neighbors is colored neither by the previous color of $T$, nor by its current color. In this case the processor "returns" this information to the root of $T$. Upon receipt of this information the root of $T$ extends its tid by a random bit which is distributed again along the edges of $T$. At the same time $T^{\prime}$ may also extend its own tid. Since each extension is done randomly, symmetry is eventually broken and the system reaches a leader configuration. A leader configuration is a configuration with exactly one leader such that in any execution that starts with this configuration the leader is fixed. Once there exists exactly one leader in the system the protocol ensures that this leader extends his tid at most once (before a safe configuration is reached).

\subsection{Formal Description of the Protocol}

The code that appears in Fig. 1, Section 3.2, below, is written for processor $P_{i}$ that has $\Delta$ neighbors. Each processor, $P_{i}$, owns a register in which it writes and all its neighbors read. The register of $P_{i}$ consist of the following fields: $t i d_{i}, \mathrm{dis}_{i}, f_{i}$, color $_{i}, a c k_{i}$ and $o t_{i}$.

$t i d_{i} \quad$ The field $t i d_{i}$ indicates the identity of the tree to which $P_{i}$ belongs in the FSG.

$d i s_{i} \quad$ The field $d i s_{i}$ indicates the distance of $P_{i}$ from the root of the tree it belongs to. In case $d i s_{i}=0, P_{i}$ is a root processor.

$f_{i} \quad$ In case $d i s_{i} \neq 0$, the value in the field $f_{i}$ indicates which of the neighbors of $P_{i}$ is its father. $f_{i}$ holds the index of the link of $P_{i}$ that connect $P_{i}$ with its father. Thus, the neighbor of $P_{i}$ that is connected to $P_{i}$ through this link can determine by the value of $f_{i}$ 
and $d i s_{i}$ that $P_{i}$ is its son ${ }^{3}$.

Color For the execution of the global synchronization protocol, as we describe in the sequel, we use the color field. color may contain eight colors which are denoted by integers of values 0 to 7 .

Ack The ack field is also used for the global synchronization protocol (below). ack is a boolean field.

Ot The field ot is assigned by true whenever a processor notes that one of its neighbors has color that is different from both its own current color and the new color of its father.

Each time $P_{i}$ reads the value of $f_{j}$, dis $s_{j}$, tid $_{j}$, color $_{j}, a c k_{j}$, ot ${ }_{j}$ of its neighbor $P_{j}, P_{i}$ assigns those values in its internal variables $f_{i}[j], \operatorname{dis}_{i}[j], \operatorname{tid}_{i}[j], \operatorname{color}_{i}[j], \operatorname{ack}_{i}[j]$, and ${ } t_{i}[j]$, respectively.

The function extend-tid chooses a random bit and concatenates it to the tail of the current tid. The function $\operatorname{son}(j)$ executed by $P_{i}$ is true only when $P_{j}$ is the son of $P_{i}$, this is indicated by the value of $f_{i}[j]$. The function choose_color chooses randomly between six (out of the possible eight) colors that are not equal to the previous_color and to (the current) color and assigns previous_color by the value of (the current) color.

We define the lexicographic order between two values of tid fields as follows: Let $t i d_{1}$ and $t i d_{2}$ be two values of tid fields. The relation $t i d_{1}>t i d_{2}$ is true when $t i d_{2}$ is a prefix of $t i d_{1}$, or (when neither $t i d_{1}$ nor $t i d_{2}$ is a prefix of the other) if $t i d_{1}^{\prime}$ and $t i d_{2}^{\prime}$ are derived from $t i d_{1}$ and $t i d_{2}$ by removing their common maximal prefix and the first bit of $t i d_{1}^{\prime}$ is 1 . Define the relation $\succ$ over pair of processors fields ( $t i d$, dis) as follows: $\left(\left(t i d_{1}, d i s_{1}\right) \succ\left(t i d_{2}, d i s_{2}\right)\right)$ if $t i d_{1}>t i d_{2}$ (as defined above) or when $t i d_{1}=t i d_{2}$, then $d i s_{1}<d i s_{2}$. In such a case we say that the pair $\left(t i d_{1}, d i s_{1}\right)$ is greater than $\left(t i d_{2}, d i s_{2}\right)$.

\subsubsection{Description of the Code}

The code of the protocol appears in Fig. 1 and Fig. 2. The code consists of a single infinite do forever loop, the lines of this loop are described below.

Line $2-P_{i}$ reads the registers of its neighbors.

Lines 3 to 10 - Using the values read, $P_{i}$ calculates the maximal tid among the tids of its neighbors. Then $P_{i}$ finds the minimal dis of a neighbor among the neighbors holding the maximal tid. At last $P_{i}$ finds the index of the first neighbor holding the above maximal tid and minimal dis and updates the local variables $F$, $C, A$, and OT accordingly.

Line 11 to 13 - If $P_{i}$ finds that it has no neighbor $P_{j}$ with $\left(t i d_{j}, d i s_{j}\right) \succ\left(t_{i d}, d i s_{i}\right)$ then $P_{i}$ becomes a root. Otherwise, $P_{i}$ updates the values of its tid and dis according to the values it reads.

Line 14 - When $P_{i}$ finds that for every neighbor it holds that $\operatorname{tid}_{i}=\operatorname{tid}_{j}$ and $\left|d i s_{i}-d i s_{j}\right| \leq 1, P_{i}$ assumes that the cycle elimination is over and joins trees fusion.

3. It is assumed that $P_{i}$ knows the indices given by its neighbors to every of its links.

1 do forever

$\left({ }^{*}\right.$ Reading $\left.*\right)$

2 for $j:=1$ to $\Delta$ do $\left(\operatorname{tid}_{i}[j], \operatorname{dis}_{i}[j], f_{i}[j], \operatorname{color}_{i}[j], \operatorname{ack}_{i}[j], o t_{i}[j]\right)$ $:=\operatorname{read}\left(r_{j}\right)$;

3 max_tid $:=\max \left(\operatorname{tid}_{i}[j]\right)$;

$4 \min \_$dis $:=\min \left\{\operatorname{dis}_{i}[j] \mid \operatorname{tid}_{i}[j]=\max \_t i d\right\}$;

5 if $\left(\operatorname{tid}_{i}\left[f_{i}\right], \operatorname{dis}_{i}\left[f_{i}\right]\right)=($ max_tid, min_dis $)$ then

6 else

7 begin

$8 \quad F:=\left\{\right.$ first $j \mid$ tid $_{i}[j]=\max \_t i d$, dis $_{i}[j]$ $=$ min - dis $\}$;

$9 \quad(C, A, O T):=\left(\right.$ color $_{i}[F]$, false, false $)$;

10 end

( Cycles elimination *)

11 if $\left(t i d_{i}, d i s_{i}\right) \succeq($ max_tid, min_dis $)$

12 then write $\left(\right.$ dis $\left._{i}, f_{i}\right):=(0$, nil $)\left({ }^{*}\right.$ become a root $\left.{ }^{*}\right)$

13 else write $\left(\right.$ tid $_{i}$, dis $_{i}, f_{i}$, color $_{i}$, ack $_{i}$, ot $\left._{i}\right)$ $:=($ max_tid, min_dis $+1, F, C, A, O T)$;

14 if $\left\{\forall j \mid\left(t i d_{i}[j]=t i d_{i}\right)\right.$ and $\left(\left|d i s_{i}-d i s_{i}[j]\right| \leq 1\right)$ then tree-fusion;

15 end

Fig. 1. Leader election in general graph systems.

16 Procedure tree-fusion

$\left({ }^{*} \operatorname{Root}^{*}\right)$

17 if $\left(\right.$ dis $\left._{i}=0\right)$ and $\left\{\forall j \mid \operatorname{son}(j) \Rightarrow\left(\left(\right.\right.\right.$ color $_{i}[j]=$ color $\left._{i}\right)$ and $\left.\left.\operatorname{ack}_{i}[j]\right)\right\}$ then

18

19

20

21

22

23

24

25

26

27

28

29

30

31

32

33

34

35 end

Fig. 2. Procedure tree_fusion.

Lines 17 to 22 - These lines consider the case in which $P_{i}$ is a root and discovers that it finished coloring its tree with a single color. In this case $P_{i}$ checks whether any processor of its tree detected the existence of another tree. If the existence of such a tree is detected $P_{i}$ extends its $t i d$. Then $P_{i}$ starts coloring its tree with a new color. 
Lines 23 to 29 - These lines consider the case in which $P_{i}$ is not a root and discovers that its father is colored with a new color. If $P_{i}$ finds that it has a neighbor $P_{j}$ for which color $_{j} \notin\left\{\right.$ color $_{i}$, color $\left._{i}[f]\right\}$ it concludes that $P_{j}$ belongs to another tree. $P_{i}$ writes its conclusion in $o t_{i}$ and then sets color $r_{i}$ to the color of its father.

Lines 30 to 34 - These lines consider the case in which $P_{i}$ is not a root and discovers that its sons finished coloring their subtrees with the color of color ${ }_{i} . P_{i}$ collects the indications on the existence of other trees and report to its father that it finished coloring its subtree.

\subsection{Correctness and Complexity Proofs}

Toward proving the correctness of the protocol we first describe the synchronization building blocks used by the protocol. Then we prove that the system reaches a configuration after which there is a single tree with a leader. The proof is completed by showing that this tree is fixed.

\subsubsection{Synchronization}

In this subsection we present two self-stabilizing synchronization protocols: a local synchronization protocol and a global-synchronization protocol. The local-synchronization protocol is designed for a two processor system. The globalsynchronization protocol is an extension of the localsynchronization protocol and is designed for a tree structured system. These protocols are used as components in a protocol for leader election in general graphs. Procedure tree-fusion in Fig. 2 uses a global synchronization protocol. Nevertheless, both synchronization protocols are of an independent interest. The global-synchronization protocol, that stabilizes in $O(\Delta \mathcal{D})$ rounds, may be used as an efficient self-stabilizing synchronizer that implements selfstabilizing synchronous protocols in an asynchronous system. The global-synchronization protocol can also be used as an efficient self-stabilizing snapshot and reset protocol (see [26], [5]).

\subsubsection{Self-Stabilizing Local-Synchronization Protocol}

A local-synchronization protocol is designed for a system that consists of two processors $P_{f}$ and $P_{s}$ and two registers color $_{f}$ and color.$P_{f}\left(P_{s}\right)$ writes in color ${ }_{f}\left(\right.$ color $\left._{s}\right)$ and reads from color $_{s}\left(\right.$ color $\left._{f}\right)$. The color of $P_{f}\left(P_{s}\right)$ is the value stored in color $\left(\right.$ color $\left._{s}\right)$, respectively.

Informally the task of the local-synchronization protocol is to ensure that $P_{f}$ changes its color infinitely often and to ensure that following every time that $P_{f}$ changes its color $P_{s}$ changes its color to the color of $P_{f}$ and only then $P_{f}$ changes its color again. More precisely the task of the localsynchronization protocol is defined by a set of executions in which:

- $P_{f}$ changes the value of color $_{f}$ infinitely often.

- Immediately before any change of the value of color $f$ it holds that color $_{f}=$ color $_{s}$.

- Immediately after any change of the value of color $_{s}$ it holds that color $_{s}=$ color $_{f}$.

The local-synchronization protocol is defined below. During the execution of the protocol whenever $P_{f}\left(P_{s}\right)$ reads the value of color $_{s}$ it assigns this value in $\operatorname{color}_{f}[s]\left(\operatorname{color}_{s}[f]\right.$, respectively). Therefore, the value of $\operatorname{color}_{f}[s]\left(\operatorname{color}_{s}[f]\right)$ is the value obtained in the last time, if any, $P_{f}\left(P_{s}\right)$ read color $\left(\operatorname{color}_{f}[s]\right.$, respectively). The protocol uses three colors (or more). $P_{f}$ uses the function choose-color which always selects a color that is different from both the current color and the previous color of $P_{f}$. To do so $P_{f}$ has an internal variable called previous-color. Every time the choose-color function is executed it chooses a color that is equal neither to previous_color nor to color $_{f}$ then assigns previous_color $:=$ color $_{f}$ and at last returns the color that it chooses.

Program of $P_{f}-P_{f}$ repeatedly executes:

(lf1) reads color $r_{s}$ into color $[s]$ and

(lf2) if color $_{f}[\mathrm{~s}]=$ color $_{f}$ then it assigns color $_{f}:=$ choose-color(previous-color, color $_{f}$ ).

Program of $P_{s}-P_{s}$ repeatedly executes:

(ls1) reads color into color $[f]$ and

(ls2) if color $_{s}[f] \neq$ color $_{s}$ then it assigns color ${ }_{s}:=$ color $_{s}[f]$.

In the following lemma we show that any execution of the local synchronization protocol stabilizes after $P_{s}$ executes a constant number of atomic steps. The lemma uses the following definition which will be used throughout the paper.

DEFINITION 1. Let $Q$ be a program that consists of exactly one infinite do forever loop such that the first (last) line of $Q$ is the first (last) line of this loop. A processor that executes $Q$ completes a loop iteration during an execution $E$ if it executes the first line of the loop during one atomic step of $E$ and executes the last line of the loop in a later atomic step of that execution.

Let $l$ be a bound on the number of atomic steps executed during arbitrary execution $E$, that starts with an atomic step in which the processor executes the first line of the loop of $Q$ and ends with the first successive execution of the last line of the loop of $Q$. By the nature of self-stabilizing protocols a processor might start with any atomic step in the loop of $Q$. Thus, the number of atomic steps needed to complete a loop iteration is bounded by $2 l-1$.

The next Lemma uses $k$ (and not 2) for the number of atomic steps needed to complete a loop iteration by $P_{s}$. This choice prepares the way for future reasoning.

LEMMA 1. If $k$ is the number of atomic steps needed to complete a loop iteration by $P_{s}$ then after at most $4 k$ atomic steps of $P_{s}$ the local synchronization protocol is stabilized.

PROOF. First we observe that a configuration in which color $_{f}$ $=$ color $_{s}[f]=$ color $_{s}$ is a safe configuration for the local synchronization protocol. Once such a configuration is reached and until $P_{f}$ changes the value of color,$P_{s}$ cannot change the value of color $_{s}[f]$ and color ${ }_{s}$. When $P_{f}$ changes the value of color $_{f}, P_{f}$ does not change the value of color $_{f}$ again before the system reaches a configuration $c^{\prime}$ in which color $_{f}=\operatorname{color}_{s}[f]=$ color $_{s}$, and so forth. Thus, it is sufficient to prove that such a configuration is reached within $4 k$ atomic steps of $P_{s}$.

First we assume that color $_{f}$ is not changed during $k$ successive atomic steps of $P_{s}$. In this case during these $k$ atomic steps $P_{s}$ reads color $_{f}$ and writes this color in color $_{s}$. Thus, a configuration in which color $_{s}=$ color $_{s}[f]=$ color $_{f}$ is reached and the system is stabilized. 
Now consider executions in which color $_{f}$ is changed every at most $k$ atomic steps of $P_{s}$. Consider such execution, $E$, that contains 4 successive changes of color $_{f}$. Let color ${ }^{i}$ be the color assigned to color $_{f}$ in the $i$ th color change of color. Note that $E$ contains at most $4 k$ atomic steps of $P_{s}$. E contains the following steps, in the specified order:

1) $P_{f}$ writes color ${ }^{1}$ to color $_{f}$

2) $P_{f}$ reads color in color

3) $P_{f}$ writes color ${ }^{2}$ to color

4) $P_{f}$ reads color ${ }^{2}$ in color

5) $P_{f}$ writes color ${ }^{3}$ to color $_{f}$

6) $P_{f}$ reads color ${ }^{3}$ in color

By the programs of $P_{f}$ and $P_{s}$ it holds that: Between Step 2 and Step 4, $P_{s}$ must write color ${ }^{2}$ in color ${ }_{s}$ and between Step 4 and Step 6, $P_{s}$ must write color ${ }^{3}$ in color ${ }_{s}$. Hence, between Step 2 and Step 6, $P_{s}$ must read $\mathrm{color}^{3}$ in color. Since color ${ }^{1}$, color $^{2}$, color $^{3}$ are distinct, that read operation of $P_{s}$ must occur after Step 5 (and before Step 6). Thus, between Step 5 and Step 6, $P_{s}$ reads color ${ }^{3}$ in color $r_{f}$ and then writes color ${ }^{3}$ in color $_{s}$. Immediately after this write operation, $\operatorname{color}_{f}=\operatorname{color}_{s}[f]=$ color $_{s}$ as required.

\subsubsection{Self-Stabilizing Global-Synchronization Protocol}

A directed graph is an in-tree if the undirected underlying graph is a tree, and if every edge of the tree is directed towards a common root. For the sake of readability we use the term tree instead of in-tree. A global synchronization protocol is a protocol for a tree structured system, with a root $P_{r}$. The global-synchronization protocol uses two fields of a register for each processor. A processor, $P_{i}$, writes in two fields called color ${ }_{i}$ and $a c k_{i}$ that are read by any of its neighbors in the tree.

Informally the task of a global-synchronization protocol is to ensure that $P_{r}$ changes its color infinitely often. Following every time that $P_{r}$ changes its color all the processors in the tree change their color to the color of $P_{r}$ and only then $P_{r}$ changes its color again. More precisely the task of a global-synchronization protocol is defined by a set of executions in which:

1. $P_{r}$ changes the value of color $_{r}$ infinitely often.

2. For any processor $P_{i}$, immediately before any change of the value of color $_{r}$ it holds that color ${ }_{i}=$ color $_{r}$.

3. For any processor $P_{i}$, immediately after any change of the value of color $_{i}$ it holds that color $_{i}=$ color $_{r}$.

The global-synchronization protocol is defined below. During the execution of the protocol whenever $P_{i}$ reads the value of $\operatorname{color}_{j}\left(a c k_{j}\right)$ it assigns this value in $\operatorname{color}_{i}[j]\left(a c k_{i}[j]\right.$, respectively). To determine a new color $P_{r}$ uses the choose-color function that was defined above.

Program of $P_{r}-P_{r}$ repeatedly executes:

(gr1) Reads the fields color ${ }_{i}$ and $a c k_{i}$ of each of its sons $P_{i}$ and

(gr2) If for every son it holds that color $_{r}[i]=$ color $_{r}$ and $a c k_{r}[i]=$ true then it sets color $_{r}:=$ choose-color (previous-color, color ${ }_{r}$ ).

Program of $P_{i} i \neq r$-Let $P_{f}$ be the father of $P_{i}$ in the tree. $P_{i}$ repeatedly executes: (gi1) Reads the field color $_{f}$ of its father and the fields color and $a c k_{s}$ of each of its sons $P_{s}$, and

(gi2) If color $i f] \neq$ color $_{i}$ then $P_{i}$ assigns color $r_{i}:=$ color $_{i}[f]$ and $a c k_{i}:=$ false, otherwise

(gi3) If for each of its sons, $P_{s}, \operatorname{color}_{i}[s]=$ color $_{i}$ and $a c k_{i}[s]$ $=$ true, then $P_{i}$ assigns ack $:=$ true.

LEMMA 2. For any tree of depth $\mathcal{D}$, in which $\Delta$ is the maximal degree of a node, the global-synchronization protocol stabilizes within $O(\Delta \mathcal{D})$ rounds.

PROOF. The program of each processor in the globalsynchronization protocol consists of one infinite do forever loop. There is a constant $k_{1}$ such that a loop iteration of a processor with $\Delta$ neighbors in the tree is completed within $k_{1} \Delta$ atomic steps. Hence $k_{1} \Delta$ is an upper bound on the number of atomic steps needed for a processor to complete at least one loop iteration of the global synchronization protocol.

First we show that requirement 1 of a global synchronization protocol holds. In fact we show that $P_{r}$ changes its color at least once every $2 k_{1} \Delta \mathcal{D}+k_{1} \Delta$ rounds: Assume that color is not changed during an execution $E$ of $2 k_{1} \Delta \mathcal{D}+k_{1} \Delta$ rounds. Let $P_{i}$ be a neighbor of $P_{r}$. During the first $k_{1} \Delta$ rounds of $E P_{i}$ executes (gi1) and then (gi2) at least once. Immediately after $P_{i}$ executes (gi1) and (gi2) it holds that color ${ }_{i}=$ color $_{i}[r]=$ color $_{r}$ (the color of $P_{i}\left(\right.$ color $\left._{i}\right)$ is equal to the color $P_{i}$

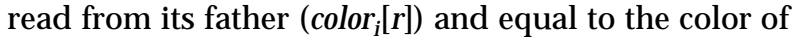
its father $\left(\right.$ color $\left._{r}\right)$ ). Any further execution of (gi1), (gi2) or (gi3) does not change the color of color ${ }_{i}$. Since $P_{i}$ is an arbitrary neighbor this equation hold for every neighbor of $P_{r}$ while $P_{r}$ does not change its color. The same arguments holds during the second $k_{1} \Delta$ rounds of $E$ for processors that are in distance two from $P_{r}$. Continuing this way, following the $i$ th $k_{1} \Delta$ rounds of $E$ all the processors whose depth is less than or equal to $i$ read the color of $P_{r}$ from their father and are colored with the color of $P_{r}$. Thus, following $k_{1} \Delta \mathcal{D}$ rounds the entire tree is uniformly colored.

Let $E^{\prime}$ be the suffix of $E$ that follows the first $k_{1} \Delta \mathcal{D}$ rounds of $E$. During the first $k_{1} \Delta$ rounds of $E^{\prime}$ every leaf, $P_{i}$, executes (gi1) and then (gi3), hence sets $a c k_{i}=$ true. While the tree is uniformly colored any further execution of (gi1), (gi2) or (gi3) does not change the value of $a c k_{i}$. Hence, after the first $k_{1} \Delta$ rounds of $E^{\prime}$ $a c k_{i}=$ true for every leaf $P_{i}$. The same argument holds during the second $k_{1} \Delta$ rounds of $E^{\prime}$ for every processor $P_{i}$ whose sons are leaves. Continuing this way, following the $i$ th $k_{1} \Delta$ rounds of $E^{\prime}$ it holds that $a c k_{j}=$ true for every processor $P_{j}$, such that the subtree rooted at $P_{j}$ is of depth less than or equal to $i$. Thus, following $2 k_{1} \Delta \mathcal{D}$ rounds the entire tree is uniformly colored and for every processor $P_{i}$ in the tree, $a c k_{i}=$ true. Hence, in the next $k_{1} \Delta$ rounds $P_{r}$ executes (gr1) and (gr2) and assigns a new color to color .

Now we show that requirements 2 and 3 of a global synchronization protocol hold too. Let $E$ be an execution starting from arbitrary configuration, $c_{0}$. Let $P_{r}, P_{1}, \ldots, P_{i}, \ldots, P_{l}$ be a path from a root of a tree $P_{r}$ to a leaf in that tree, $P_{l}$. Let $E_{i}$ be a suffix of $E$ that starts immediately after $4 k_{1} \Delta i$ rounds of $E$. We prove by induction on $i$ that during $E_{i}$ it holds that: 
ASSUMPTION 1. Immediately before $P_{r}$ changes its color it holds that color $_{r}=$ color $_{1}=\ldots=$ color $_{i}$.

ASSUMPTION 2. In any configuration of $E_{i}$ that appears after $P_{i}$ changes its color for the first time (and in the same time, assigns $a c k_{i}:=$ false) it holds that if $a c k_{i}=$ false then color $_{r}=$ color $_{1}=\ldots=$ color $_{i}$.

BASE CASE, $\mathrm{I}=1$. For every father-son pair $\left(P_{r}, P_{1}\right)$, the executed protocol is the local-synchronization protocol: (gr1) and (gr2) includes (lf1) and (lf2), (gi1) and (gi2) includes (ls1) and (ls2). Thus, by Lemma 1 following $4 k_{1} \Delta$ rounds it holds that color $_{r}=$ color $_{1}$ immediately before any color change in color $_{r}$. Thus, Assumption 1 above is proven. Now we prove Assumption 2. By Lemma 1 during the first $4 k_{1} \Delta$ rounds of $E$ a configuration $c$ in which color $_{r}=$ color $_{1}[r]=$ color $_{1}$ is reached. Following $c$, color ${ }_{1}$ is not changed till color $_{r}$ is changed. Let $c^{\prime}$ be a configuration that immediately follows the first change in color $_{r}$ after $c$ i.e., $c^{\prime}$ follows an atomic step during which $P_{r}$ changes color $r_{r}$ and sets $a c k_{r}:=$ false. By the fact that the local-synchronization protocol is stabilized it holds that following $c^{\prime}$ the fatherson pair $\left(P_{r}, P_{1}\right)$ may repeatedly change their color and ack values only in the following order:
1) $P_{1}$ changes color ${ }_{1}$ to the color of color $_{r}$ and sets $a c k_{1}$ $:=$ false
2) $P_{1}$ sets $a c k_{1}:=$ true
3) $P_{r}$ changes color $r$.

Thus, following the first time $P_{1}$ executes Step 2 , above, the equation $a c k_{1}=$ false holds only during subexecutions that start immediately after Step 1 and end immediately before the next Step 2. During these subexecutions color $_{r}=$ color $_{1}$.

INDUCTION STEP. We first prove that following $4 k_{1} \Delta i$ rounds the father-son pair $\left(P_{i}, P_{i+1}\right)$ executes the local synchronization protocol:

LEMMA 3. Following $4 k_{1} \Delta i$ rounds the father-son pair $\left(P_{i}, P_{i+1}\right)$ executes the local synchronization protocol.

PROOF. To prove the lemma, it is sufficient to show that when the induction hypotheses 1 and 2 hold for $i$ then in $E_{i}$ it hold that:

1) $P_{i+1}$ repeatedly reads color $i$ and whenever color $_{i+1}[i]$ $\neq$ color $_{i+1}, P_{i+1}$ assigns color $_{i+1}:=$ color $_{i}$.

2) between any two changes of color $_{i}, P_{i}$ reads color $_{i+1}$ and finds color $i=$ color $_{i}[i+1]$.

3) the sequence of colors that is assigned to color $_{i}$ is equal to the sequence of colors that is assigned to color $r$.

(gi1) and (gi2) of the global synchronization protocol imply 1 . To prove 2 , we show that during $E_{i} P_{i}$ assigns $a c k_{i}:=$ true between any two successive changes in the value of color ${ }_{i}$. Whenever $P_{i}$ changes its color it sets $a c k_{i}:=$ false. By Assumption 2 while $a c k_{i}=$ false it holds that color $_{r}=$ color $_{1}=\ldots=$ color $_{i}$. Hence $P_{i}$ does not change its color thereafter before it assigns $a c k_{i}:=$ true. This latter operation is done only after $P_{i}$ finds that color $_{i}[i+1]=$ color $_{i}$, as required by the local synchronization protocol. This proves 2, above.

By Assumption 1, between any two changes in col$o r_{r}$, there is a configuration in which color $_{i}=$ color $_{r}$. By
Assumption 2, and the fact that whenever $P_{i}$ assigns a color to color $r_{i}$ it sets $a c k_{i}=$ false, it holds that color $r_{i}$ is always changed to the color of color $r$. Hence, 3.

By Lemma 3 and by Lemma 1 it must hold that in the first $4 k_{1} \Delta$ rounds of $E_{i}$ a configuration $c$ is reached in which color $_{i}=$ color $_{i+1}[i]=$ color $_{i+1}$. Hence, the local synchronization protocol executed by $P_{i}$ and $P_{i+1}$ is stabilized during those $4 k_{1} \Delta$ rounds. Thus, following $c$ the father-son pair $\left(P_{i}, P_{i+1}\right)$ may repeatedly change their color and ack values only in the following cyclic order:

1) $P_{i}$ changes color $r_{i}$ and sets $a c k_{i}:=$ false

2) $P_{i+1}$ changes color $r_{i+1}$ to the color of color $_{i}$ and sets $a c k_{i+1}$ $:=$ false

3) $P_{i+1}$ sets $a c k_{i+1}:=$ true

4) $\mathrm{P}_{\mathrm{i}}$ sets ack $:=$ true.

We now prove that following $c$ the induction assumptions hold for $i+1$ :

ASSUMPTION 1. We claim that following $c$, color $_{r}$ is changed only when color $_{i}=$ color $_{i+1}$. Let $c^{\prime}$ be the configuration that follows $c$ and immediately precedes the atomic step in which $P_{r}$ changes the color of color $r$. Assume toward a contradiction that in $c^{\prime}$ color $_{i} \neq$ color $_{i+1}$. Then $P_{i}$ changed its color at least once after $c$. Consider the last such change before $c^{\prime}$. We now show that $a c k_{i}$ must be false in $c^{\prime}$. ack is true only during subexecutions that start immediately after 4 and end immediately before the next 1 . During these subexecutions color $_{i}=$ color $_{i+1}$, and hence $a c k_{i}=$ false in $c^{\prime}$. Thus, by Assumption 2 color $_{r}$ is not changed. Hence there is a contradiction.

ASSUMPTION 2. First, we show that whenever $a c k_{i+1}=$ false then $a c k_{i}=$ false, then we apply the induction assumption on $a c k_{i}$ : following $c P_{i}$ and $P_{i+1}$ change the value of color $_{i}$, color $_{i+1}$ and $a c k_{i}, a^{a c k_{i+1}}$, according to Steps 1 to 4 , above. $P_{i+1}$ changes its color, according to 2 above, only after $P_{i}$ executes step 1 in which $P_{i}$ changes its color and sets $a c k_{i}=$ false. After executing $1, P_{i}$ does not change the value of $a c k_{i}$ to true unless color $_{i}=$ color $_{i+1}$ and $a c k_{i+1}=$ true. Thus, during any subexecution that starts immediately after 1 and ends immediately after the next $2, a c k_{i}=$ false. $a c k_{i+1}=$ false only during subexecutions that start immediately after 2 and ends immediately before the next 3 . During these subexecutions $a c k_{i}=$ false and color $_{i}=$ color $_{i+1}$. Now we complete the proof by the use of the induction assumption applied to $P_{i}$ : if $a c k_{i}$ $=$ false then $_{\text {color }}=$ color $_{1}=\ldots=$ color $_{i}$.

\subsubsection{Single Tree With a Leader}

First we prove that in every fair execution eventually FSG becomes a forest and once this happens FSG remains a forest for the rest of the execution. Then we prove that FSG is converted to a single tree and in the end we prove that this tree is fixed forever.

LEMMA 4. For any processor $P_{i}$ and any execution $E$ the value of $\left(\right.$ tid $_{i}$, dis $\left._{i}\right)$ does not decrease during $E$.

PROOF. The value of $\left(t i d_{i}, d i s_{i}\right)$ is changed only in lines 12 , 13, 20 of Fig. 1 and Fig. 2. It is easy to see that when either line 12 or 20 is executed, the value of $\left(t i d_{i}, d i s_{i}\right)$ 
does not decrease. By the nature of read write atomicity line 13 is included in the same atomic step with the test of line 11 . This test ensures that $\left(t i d_{i}, d i s_{i}\right)$ never decreases.

\section{DEFINITION 2.}

1) A processor completes a loop iteration during an execution if it executes line 2 and then executes lines 3 to 15 of the protocol.

2) A processor completes a read iteration during some execution of our protocol if it executes line 2 and then lines 3 to 10 of the protocol.

As described before, an edge $\left(P_{f} P_{s}\right)$ belongs to FSG $(c)$ if in configuration $c, f_{s}$ points to $P_{f}$ and $d i s_{s}>0$. In this case we say that $\left(P_{f}, P_{s}\right)$ is a father-son pair in $c$.

LEMMA 5. Let $E^{\prime}=\left(c_{0}, \ldots, c\right)$ be (a prefix of) an execution of the protocol in which each processor completes a loop iteration. The following hold for any father-son pair $\left(P_{f}, P_{s}\right)$ in $c$ :

1) Following the first read iteration in $E^{\prime}, P_{s}$ executes the write operation in line 13 at least once.

2) Let $c_{i}$ be the configuration reached by the system immediately after $P_{s}$ executes the write operation of line 13 for the last time during $E^{\prime}$. In $c_{i}$ the following relation holds:

$$
\left(t i d_{f}, d i s_{f}\right) \succeq\left(t i d_{s}[f], d i s_{s}[f]\right) \succ\left(t i d_{s}, d i s_{s}\right)
$$

ProOF. Since $P_{s}$ completes a loop iteration, it executes the if statement (Line 11) at least once before $c$ is reached. Within this if-statement $P_{s}$ either declares itself a root (Line 12) or declares some processor as its father (Line 13). Consider the last time this if-statement is executed before $c$ : In $F S G(c), P_{s}$ is the son of $P_{f}$, therefore in the last time line 11 was executed $P_{s}$ chose to execute line 13. This proves 1 .

The value of $t i d_{s}[f], d i s_{s}[f]$ in $c_{i}$, is obtained by reading $\left(t i d_{f}, d i s_{f}\right)$ (in line 2$)$ at an earlier step. By Lemma 4 $\left(t i d_{f}, d i s_{f}\right)$ does not decrease, hence the leftmost relation holds. Configuration $c_{i}$ immediately follows the last execution of Line 13 by $P_{s}$, in which $P_{s}$ declares $P_{f}$ to be its father. This is done by $P_{s}$ writing $\left(t i d_{s}[f], \operatorname{dis}_{s}[f]\right.$ $+1)$ in $\left(t i d_{s}, d i s_{s}\right)$, which implies the rightmost relation, hence 2 is proven.

LEMMA 6. Let $E^{\prime}=\left(c_{0}, \ldots, c\right)$ be an execution in which each processor completes a loop iteration. For every father-son pair $\left(P_{f}, P_{s}\right)$ in $c$, relation $\left(^{*}\right)$ holds

PROOF. Consider an arbitrary father-son pair $\left(P_{f}, P_{s}\right)$. Let $c_{i}$ be the configuration reached after the last time $P_{s}$ executes the if statement of line 11 before $c$. By Lemma 5, the relation $\left(^{*}\right)$ holds in $c_{i}$. To complete the proof we show that the relation $\left(^{*}\right)$ is preserved during $E^{\prime \prime}=\left(c_{i}\right.$, $\left.c_{i+1}, \ldots, c\right)$. Throughout $E^{\prime \prime}, P_{s}$ is the son of $P_{f}$ hence it is not a root. The only instruction in which a nonroot processor changes its (tid, dis) is the if statement of line 11. Since $c_{i}$ follows the last time $P_{s}$ executes this if statement, $\left(t i d_{s}, d i s_{s}\right)$ is constant and $P_{f}$ is the father of $P_{s}$ throughout $E^{\prime \prime}$. To complete the proof we observe that $\left(t i d_{s}[f], d i s_{s}[f]\right)$ is updated by $P_{s}$ copying $\left(t i d_{f}, d i s_{f}\right)$. By Lemma 4 this pair only grows, hence the proof.
LEMMA 7. If $c$ is a configuration in which the relation $\left({ }^{*}\right)$ holds for every father-son pair then $F S G(c)$ is a forest of trees.

PROOF. To prove the lemma we have to show that the outdegree of each node is at most 1 and the underlying graph has no cycles. Each nonroot processor has at most one father (defined by the value of $f$ ). By Lemma 6 the relation $\left(^{*}\right)$ holds for every father-son pair in $F S G(c)$, hence $F S G(c)$ contains no cycles.

DEFINITION 3. Configuration, $c$, is a forest configuration if the relation $\left.{ }^{*}\right)$ holds for every father-son pair $\left(P_{f}, P_{s}\right)$ in $c$, and for every pair of neighbors $P_{i}$ and $P_{j}\left(t i d_{i}, d i s_{i}\right) \geq$ $\left(t i d_{j}[i], d i s_{j}[i]\right)$.

COROLLARY 8. In every execution E:

1) A forest configuration is reached following $O(\Delta)$ rounds.

2) If $c_{i}$ is a forest configuration and $c_{i} \rightarrow c_{i+1}$ then $c_{i+1}$ is a forest configuration.

ProOF. By Lemma 6, a forest configuration is reached in every execution after each processor has completed at least a single loop iteration. By the definition of loop iteration and by the code there exists a constant $k_{1}$ such that processor $P_{i}$ with $\Delta$ neighbors takes $k_{1} \Delta$ steps to complete a loop iteration. Hence in any execution, the system reaches a forest configuration within $O(\Delta)$ rounds which proves 1 . The proof of 2 is by arguments similar to the arguments of Lemma 6 .

DEFINITION 4. $R(c)$ is the set of the root processors in configuration $c$.

LEMMA 9. For any two forest configurations, $c_{i}, c_{i+1}$, such that $c_{i+1}$ is reached by an arbitrary atomic step a from $c_{i}, R\left(c_{i}\right) \supseteq R\left(c_{i+1}\right)$.

PROOF. Assume in contradiction that during $a$ there is a nonroot processor $P_{s}$ that assigns 0 in $\operatorname{dis}_{s}$. Let $P_{f}$ be the father of $P_{s}$ just before the execution of $a$. Since $a$ must contain a write operation of line $12 a$ starts by the execution of line 3 to 11 by the processor $P_{s}$, when $P_{s}$ executes line $11 P_{s}$ finds that the condition $\left(t i d_{s}, d i s_{s}\right)$ $\succeq$ (max_tid, min_dis) is true, and $a$ ends with $P_{s}$ executing the atomic write of line 12. During the execution of $a$ following the execution of lines 3 to 10 it holds that (max_tid, min_dis) $\geq\left(t i d_{s}[f], d i s_{s}[f]\right)$. By the definition of forest configurations the relation $\left(^{*}\right)$ holds in $c_{i}$. Thus, (max_tid, min_dis) $\succ\left(t i d_{s}, d i s_{s}\right)$ and hence the write operation of line 12 is not executed, contradiction.

Lemma 7, Corollary 8, and Lemma 9 show that if $E$ is an execution that starts with a forest configuration, then $F S G(c)$ is a forest in every configuration $c$ of $E$ and no processor becomes a root during $E$. In the following lemmas we show that when an execution starts with a forest configuration the number of roots decreases to one in $O(\Delta \mathcal{D} \log n)$ expected number of rounds. Where $\mathcal{D}$ is the diameter of the communication graph. The following definitions are used in the sequel:

\section{DEFINITION 5.}

1) In any configuration $c, t_{i d}(c)$ is the tid of $P_{i}$ in $c . f_{i}(c)$, dis $_{i}(c)$ and the other value of $c$ are defined similarly.

2) In any configuration $c, M T I D(c)=\max \left\{\right.$ tid $\left._{i} \mid 1 \leq i \leq n\right\}$ in $c$. 
3) A uniform tid configuration $c$, is a configuration in which for every processor $P_{i}$ tid ${ }_{i}=M T I D(c)$ and for every neighbor of $P_{i}, P_{j}, t i d_{i}[j]=M T I D(c)$.

LEMMA 10. If MTID is not changed during an execution that starts with a forest configuration and contains at least $k_{1} \Delta(\mathcal{D}+1)$ rounds then a uniform tid configuration is reached.

PROOF. Let $E=\left(c_{0}, c_{1}, \ldots\right)$ be an execution that contains at least $k_{1} \Delta(\mathcal{D}+1)$ rounds throughout which MTID remains constant and in which $c_{0}$ is a forest configuration. Since $c_{0}$ is a forest configuration, there exists a non empty set, denoted $R_{m}\left(c_{0}\right)$, of root processors in $c_{0}$ whose tid is equal to $\operatorname{MTID}\left(c_{0}\right)$. By Lemma 4, the tid of a processor never decreases, on the other hand MTID remains constant throughout $E$. Therefore once a processor's tid becomes equal to $\operatorname{MTID}\left(c_{0}\right)$ it remains constant throughout $E$.

To prove the lemma we first show that during the first $k_{1} \Delta \mathcal{D}$ rounds of $E$, the tid of every processor in the system becomes equal to $\operatorname{MTID}\left(c_{0}\right)$. This is proved by induction on $d$, the distance of a processor from (the closest processor in) $R_{m}\left(c_{0}\right)$; Where distance is measured on the communication graph. Denote the distance of $P_{i}$ from $R_{m}\left(c_{0}\right)$ by $d_{i}$. We prove that after $k_{1} \Delta d$ rounds $t i d_{i}$ of any processor $P_{i}$ in distance $d_{i} \leq d$ is equal to $\operatorname{MTID}\left(c_{0}\right)$.

INDUCTION BASE $(\mathrm{d}=0)$ : Clearly, the tid of processors in $R_{m}\left(c_{0}\right)$ is equal to $\operatorname{MTID}\left(c_{0}\right)$ throughout $E$.

INDUCTION STEP: We assume that after $k_{1} \Delta d$ rounds, $\operatorname{tid}_{i}=$ $\operatorname{MTID}\left(c_{0}\right)$ for any processor $P_{i}$ in distance $d_{i}=d$ and we prove that following the next $k_{1} \Delta$ rounds $t i d_{j}=$ $\operatorname{MTID}\left(c_{0}\right)$ for any processor $P_{j}$ in distance $d_{j}=d+1$. During the rounds $d k_{1} \Delta$ through the $(d+1)$ th $k_{1} \Delta, P_{j}$ completes at least one loop iteration. In the first such loop iteration $P_{j}$ reads the tid of a processor in distance $d$ from $R_{m}\left(c_{0}\right)$ in case $t i d_{j}<M T I D\left(c_{0}\right) P_{j}$ assigns $\operatorname{MTID}\left(c_{0}\right)$ to $t i d_{j}$. Hence, following $k_{1} \Delta(d+1)$ rounds the induction hypothesis holds for every processor $P_{j}$ in distance $d_{i}\left(c_{0}\right)=d+1$.

We proved that following the first $k_{1} \Delta \mathcal{D}$ rounds of $E$ tid $=\operatorname{MTID}\left(c_{0}\right)$ for every processor. During the following $k_{1} \Delta$ rounds each processor reads the tid of every neighbor hence for every $j t i d_{i}[j]:=\operatorname{MTID}\left(c_{0}\right)$.

LEMMA 11. Let $E=\left(c_{0}, \ldots, c\right)$ be an arbitrary execution of at least $2 k_{1} \Delta \mathcal{D}$ rounds that is started with a uniform tid forest configuration and during which MTID is not changed. The following assertions hold for every configuration $c^{\prime}$ in the suffix of $E, E^{\prime}$, that starts following the first $2 k_{1} \Delta D$ rounds of $E$ :

1) $R\left(c_{0}\right)=R\left(c^{\prime}\right)$.

2) for any processor $P_{j}, \operatorname{dis}_{j}\left(c^{\prime}\right)=d_{j}\left(c^{\prime}\right)$, where $d_{j}\left(c^{\prime}\right)$ is the distance of $P_{j}$ to a closest root in $c^{\prime}$.

3) $f_{j}\left(c_{0}^{\prime}\right)=f_{j}\left(c^{\prime}\right)$ and the value of $f_{j}\left(c^{\prime}\right)$ is the index of a neighbor which is on a shortest path to one of the closest roots in $R\left(c^{\prime}\right)$.
PROOF. By Lemma 9 and the fact that $c_{0}$ is a forest configuration, the set of roots may only be decreased following $c_{0}$. Thus, to prove Assertion 1 we only have to show that during $E$ a root processor does not become nonroot. By the fact that MTID is not changed during $E$ it holds that every configuration $c$ in $E$ is a uniform tid configuration. Thus, whenever a root, $P_{i}$, calculates max_tid it assigns max_tid: = MTID. A root processor, $P_{i}$, becomes nonroot only if max_tid $>t i d_{i}$. Since $t i d_{i}=$ MTID throughout $E$ Assertion 1 holds.

Assertions 2 and 3 are implied by the following stronger claim which is proved by induction on $d$ the distance of a processor from $R\left(c_{0}\right)$ : In any configuration $c^{\prime}$ that appears following the first $2 k_{1} \Delta d$ rounds of $E$,

1) For any processor $P_{j}$ with distance

$$
d_{j}\left(c_{0}\right) \leq d \quad \operatorname{dis}_{j}\left(c^{\prime}\right)=d_{j}\left(c_{0}\right) .
$$

2) For any processor $P_{j}$ with distance $d_{j}\left(c_{0}\right) \leq d$ the value of $f_{j}\left(c^{\prime}\right)$ is not changed throughout $E^{\prime}$. The value of $f_{j}\left(c^{\prime}\right)$ is the index of the link that connects $P_{j}$ with one of $P_{j}$ s neighbors which is on a shortest path to one of the closest roots.

3) For any processor $P_{l}$ that is in distance $d_{l}\left(c_{0}\right) \geq d$ it holds that $d i s_{l} \geq d$.

INDUCTION BASE. $d_{j}\left(c_{0}\right)=0$. The proof of Assertion 1 implies 1 and 2, above. By the fact that for every processor $P_{i}$ dis $_{i} \geq 0$ Assertion 3 holds too.

INDUCTION STEP. By the induction assumption following no more than $2 k_{1} \Delta d$ rounds of $E$, Assertions 1, 2 , and 3 hold for any processor $P_{j}$ in distance $d_{j}\left(c_{0}\right) \leq d$. Let $c_{d}$ be the configuration that immediately follows the first $2 k_{1} \Delta d$ rounds. We now prove that 1,2 , and 3 hold for $d+1,2 k_{1} \Delta$ rounds following $c_{d}$. Every processor completes a loop iteration every $k_{1} \Delta$ rounds. Thus, by assertion $3, k_{1} \Delta$ rounds following $c_{d}$ any processor $P_{k}$ that is in distance $d_{k}\left(c_{0}\right)>d+1$ assigns $d i s_{k}$ by a value that is $\geq d+1$. Following additional $k_{1} \Delta$ rounds any processor $P_{m}$ that is in distance $d_{m}\left(c_{0}\right)=d+1$ reads the values of the dis fields of all the neighbors that are in distance $d$ and then (executes line 13 in which it) assigns $d i s_{m}:=d+1$ and assigns $f_{m}$ by the right value. From this point on, $P_{m}$ finds that $\left(\operatorname{tid}_{m}\left[f_{m}\right], \operatorname{dis}_{m}\left[f_{m}\right]\right)$ $=\left(\right.$ max $\_t i d$, min $\_$dis $)$and hence does not change the value of $d i s_{m}$ or $f_{m}$ (see lines 3-5 and line 13 of the code)

DEFINITION 6. A utff-execution (uniform tid fixed forest execution) is an execution in which all the configurations are uniform tid configuration and during which FSG is a constant forest. Such that, each tree in FSG is of depth less than or equal to $\mathcal{D}$.

Let $k_{2}$ be a constant that is bigger than $k_{1}(3+1 / \mathcal{D})$. By the above two lemmas any execution that starts with a forest configuration and in which MTID is constant reaches, following $3 k_{1} \Delta \mathcal{D}+k_{1} \Delta=k_{1} \Delta \mathcal{D}(3+1 / \mathcal{D})<k_{2} \Delta$ rounds, a uniform tid fixed forest execution, abbreviated utff-execution.

To prove that a root discovers the existence of other roots with equal tid we use the global-synchronization protocol 
(described in the previous section) on every tree of the fixed forest. During a utff-execution every root executes (gr1) and (gr2) of the global synchronization protocol by executing line 2 (for (gr1)), and lines 17 and 21 (for (gr2)). A nonroot processor executes (gi1), (gi2) and (gi3) of the global synchronization protocol by executing line 2 (for (gi1)), lines 24, 28 (for (gi2)), and lines 30, 33 (for (gi3)). Thus, the global synchronization protocol is executed on every tree in the fixed forest.

COROLLARY 12. There exist constant $k_{3}$ such that if $E$ is a utffexecution and $E^{\prime}$ is the suffix of $E$ that follows the first $k_{3} \Delta \mathcal{D}$ rounds of $E$, then during $E^{\prime}$ a root processor chooses a new color only when its tree is uniformly colored.

PROOF. During any utff-execution FSG defines a fixed forest of trees (with depth less than or equal to $\mathcal{D}$ ). Thus, during any utff-execution we may apply the arguments of Lemma 2 to our protocol. The arguments of Lemma 2 consider the number of rounds needed for a processor to complete at least one loop iteration of the global synchronization protocol. By the fact that any configuration of an utff-execution is a uniform tid configuration in which the value of the dis field of every processor is the minimal distance to its root the condition (in line 14) for executing lines 17, 21, 24, 28, 30, and 33 holds. Namely, the condition for executing the global synchronization protocol. In such an execution every processor completes a loop iteration in the global synchronization protocol every $k_{1} \Delta$ rounds. Hence following $4 k_{1} \Delta \mathcal{D}=k_{3} \Delta \mathcal{D}$ rounds a root chooses a new color only when all its tree is uniformly colored.

LEMMA 13. There is a constant $k_{4}$ such that every execution that starts in a forest configuration $c$ reaches in expected number of $k_{4} \Delta \mathcal{D}$ rounds a configuration $c_{i}$ which is either a leader configuration or $\operatorname{MTID}\left(c_{i}\right)>M \operatorname{MTI}(c){ }^{4}$

PROOF. The proof is by presentation of a $(7, k \Delta \mathcal{D})$-strategy for luck by the game defined by the protocol, for some constant $k$, and by the use of Theorem 5 of [17]. The strategy of luck is as follows:

Luck waits $\left(k_{2}+k_{3}\right) \Delta \mathcal{D}$ rounds (the constant $k_{2}$ is defined right after Lemma 11). If a safe configuration is reached or a configuration $c_{i}$ for which $\operatorname{MTID}\left(c_{i}\right)>$ $\operatorname{MTID}(c)$ is reached then luck wins the sl-game. Otherwise, by Lemma 10, Lemma 11, and Corollary 12 a utff-execution is started, in which the root chooses a new color only when the tree is uniformly colored.

Furthermore, by Lemma 2, in the utff-execution that starts following these $\left(k_{2}+k_{3}\right) \Delta \mathcal{D}$ rounds every root indeed chooses a new color every at most $2 k_{1} \Delta \mathcal{D}+$ $k_{1} \Delta$ rounds.

Let $P_{1}$ and $P_{2}$ be two root processors during a utffexecution such that there are neighbors $P_{i}$ and $P_{j}$ and $P_{i}$ $\left(P_{j}\right)$ belongs to the fixed tree of $P_{1}\left(P_{2}\right)$. Once luck waited $\left(k_{2}+k_{3}\right) \Delta \mathcal{D}$ rounds the strategy of luck continues as follow: If $P_{1}\left(P_{2}\right)$ chooses a new color luck intervenes and set its new color to be in $\{0,1,2,3\}(\{4,5,6,7\}$, respectively).

4. It should be noted that MTID could grow and at the same time the length of MTID could become shorter i.e., assume that MTID $=100$ and then a root processor $\mathrm{P}_{i}$ with $t_{i d}=1$ extends its $t i d$ to hold 11.

5 . Note that every such intervention is done by fixing only the most significant bit of the color.
We now show that this strategy leads to at least one extension of the tid of either $P_{1}$ or $P_{2}$ within $O(\Delta \mathcal{D})$ rounds.

We start with any combination of colors for $P_{i}$ and $P_{j}$. W.l.o.g let $P_{i}$ be the first processor that changes its color for the fourth time i.e., changed color $r_{i}$ from its original color to color ${ }^{1}$, then to color ${ }^{2}$, and to color ${ }^{3}$, and, at last, to color ${ }^{4}$. Let $a_{l \rightarrow l+1}$ be the atomic step in which $P_{i}$ changes the color of color $i_{i}$ from color to col$o r^{l+1}$. Between any two successive color changes $a_{l \rightarrow l+1}$ and $a_{l+1 \rightarrow l+2} P_{i}$ reads color $r_{j}$ and set ot $_{i}:=$ true when color $_{i}[j] \notin\left\{\right.$ color $^{1}$, color $\left.^{2}\right\}$. If $P_{i}$ did not assign ot $_{i}:=$ true following any of those changes then it read:

- That the value of color $_{j} \in\left\{\right.$ color $^{1}$, color $\left.^{2}\right\}$, between the write operations of color ${ }^{1}$ in color ${ }_{i}$ and $a_{1 \rightarrow 2}$

- That the value of color $_{j} \in\left\{\right.$ color $^{2}$, color $\left.^{3}\right\}$, between $a_{1 \rightarrow 2}$ and $a_{2 \rightarrow 3}$

- That the value of color $_{j} \in\left\{\right.$ color $^{3}$, color $\left.^{4}\right\}$, between $a_{2 \rightarrow 3}$ and $a_{3 \rightarrow 4}$

Now we show that the above yield that $P_{j}$ changes its color. Assume towards a contradiction that color $_{j}$ is not changed: since color ${ }^{1}$, color $^{2}$, and color ${ }^{3}$ are three different colors, and since color ${ }^{2}$, color $^{3}$, and color ${ }^{4}$ are also three different colors, then by the first two reads color must be color ${ }^{2}$ however by the last two reads color $r_{j}$ must be color ${ }^{3}$, a contradiction. Thus, $P_{j}$ changed its color.

By our strategy $P_{j}$ changes its color to be in $4,5,6,7$. Thus, before the forth color change of $P_{i} o t_{i}$ is assigned by true. Thus, in our sl-game a tid of a root processor is extended in $O(\Delta \mathcal{D})$ rounds and with at most seven intervention i.e., at most four intervention for $P_{1}$ and 3 for $P_{2}$. Theorem 5 of [17] implies that if luck has an $(f, r)$ strategy then the protocol reaches a leader configuration within at most $r 2^{f}$ expected number of rounds. Thus, the expected number of rounds till MTID grows is constant number of $\Delta \mathcal{D}$ (i.e., $k_{4} \Delta \mathcal{D}$ ) rounds.

LEMMA 14. There is a constant $k_{5}$ such that every execution that starts in a forest configuration $c$ reaches in $k_{5} \Delta \mathcal{D}$ rounds a configuration $c^{\prime}$ in which for every root processor $P_{i}$ in $c^{\prime}$, $\operatorname{tid}_{i}\left(c^{\prime}\right) \geq \operatorname{MTID}(c)$.

PROOF. The proof is similar to the proof of Lemma 10.

COROLLARY 15. There is a constant $k_{6}$ such that every execution that starts in a forest configuration $c$ reaches in expected number of $k_{6} \Delta \mathcal{D}$ rounds a leader configuration $c^{\prime}$ or the tid of every root processor in $c^{\prime}$ is longer than its tid in $c$ by at least $2 \log n$ bits.

PROOF. Let $k_{5}$ be the constant of Lemma 14. First we show that during the $k_{5} \Delta \mathcal{D}$ rounds that immediately follows the growth of MTID, every $\operatorname{root} P_{i}$ such that $t_{i d_{i}} \neq$ MTID extends its tid by at least one bit. Let $c$ be a configuration that immediately follows a growth of MTID. Let $P_{i}$ be the root processor that extended its tid to $M \operatorname{TID}(c)$ immediately before $c$.

For any other root processor $P_{j} \neq P_{i}$ it holds that $\operatorname{tid}_{j}(c)<t i d_{i}(c)$. By Lemma $14, k_{5} \Delta \mathcal{D}$ rounds after $c$ the

6. Note that the other case in which $P_{j}$ is the first processor to change its color for the first time is symmetric-exchange $P_{i}$ with $P_{j}$ and $4,5,6$, 7, with 0 , $1,2,3$. 
value of the tid of any root is at least the value of $M T I D(c)$. Since our protocol guarantees that whenever a root processor finds a neighbor with greater tid it becomes nonroot processor the only way $P_{j}$ could survive as a root processor is by extending its tid.

By Lemma 13 if the execution starts with a forest configuration then either a leader configuration is reached or MTID grows in expected $k_{4} \Delta \mathcal{D}$ rounds. Therefore, every expected $\left(k_{4}+k_{5}\right) \Delta \mathcal{D}$ rounds either the system reaches a leader configuration or the tid of every root processor is extended by one bit. If a leader configuration is not reached following the first growth then during additional expected $\left(k_{4}+k_{5}\right) \Delta \mathcal{D}$ rounds either the system reaches a leader configuration or the tid of every root processor is extended by 1 more bit, and so on and so forth. The proof is completed by the fact that expectation of a sum is a sum of expectations.

The proof is completed by the following corollary:

COROLLARY 16. The system elects a leader in expected $\mathrm{O}(\Delta \mathcal{D} \log n)$ rounds.

PROOF. By Corollary 15 there exists a constant $k_{6}$ such that whenever the system starts in a forest configuration $c$ the system reaches in expected $k_{6} \Delta \mathcal{D} \log n$ rounds a configuration $c^{\prime}$ which is either a leader configuration or the tid of every root in $c^{\prime}$ is longer than its tid in $c$ by at least $2 \log n$ bits. Let $P_{i}$ and $P_{j}$ be any two arbitrary root processors in $c$. Let $b_{k}\left(b_{k}^{\prime}\right)$ be the $k$ th bit of $\operatorname{tid}_{i}\left(c^{\prime}\right)\left(t i d_{j}\left(c^{\prime}\right)\right)$ (where $b_{1}, b_{1}^{\prime}$, is the most significant bit). W.l.o.g assume that $\operatorname{tid}_{i}\left(c^{\prime}\right)$ is not longer than $\operatorname{tid}_{j}\left(c^{\prime}\right)$. By Corollary 15 in the execution that starts with $c$ and ends with $c^{\prime}$ at least $2 \log n$ last bits $b_{l}, b_{l+1}$, ..., $b_{l-1+2} \log n$ of $t i d_{i}\left(c^{\prime}\right)$ were randomly chosen. By Lemma 14 if $P_{i}$ and $P_{j}$ survives as root processors during the next $k_{5} \Delta \mathcal{D}$ rounds then it must hold that $b_{i}=b_{i}^{\prime}$ for every $l \geq i \geq l-1+2 \log n$.

The probability that $P_{i}$ choose randomly a bit, $b_{k}$, that has the same value as $b_{k}^{\prime}$ is $1 / 2$. Thus, the probability that $P_{i}$ choose randomly $2 \log n$ bits, $b_{l}, b_{l+1}, \ldots$, $b_{l-1+2} \log n$, that are equal to the $2 \log n$ bits, $b_{l}^{\prime}, b_{l+1}^{\prime}, \ldots, b_{l-1+2 \log n}^{\prime}$ of $P_{j}$ is $(1 / 2)^{2 \log n}=1 / n^{2}$. The probability that there is at least one pair $P_{i}$ and $P_{j}$ such that those bits of their tid equal is less than $n^{2} / 2 \times 1 / n^{2}$ $=1 / 2$ i.e., the number of all possible pairs multiplied by the probability that the value of the bits $b_{l}, b_{l+1}, \ldots$, $b_{l-1+2} \log n \prime$, and the bits $b_{l}^{\prime}, b_{l+1}^{\prime}, \ldots, b_{l-1+2 \log n}^{\prime}$ is equal for any given pair of root processors.

Hence, the probability to reach a safe configuration following expected $k_{6} \Delta \mathcal{D} \log n+k_{5} \Delta \mathcal{D}$ rounds is greater than $1 / 2$. Similarly, the probability to reach a safe configuration following $l\left(k_{6} \Delta \mathcal{D} \log n\right)+k_{5} \Delta \mathcal{D}$ rounds is greater than $\left(1-(1 / 2)^{l}\right)$. Thus, the expected number of rounds until a leader configuration is reached is less than

$$
\begin{aligned}
& k_{5} \Delta \mathcal{D}+\sum_{l=1}^{\infty} l\left(k_{6} \Delta \mathcal{D} \log n\right)(1 / 2)^{l-1}(1 / 2) \\
= & k_{5} \Delta \mathcal{D}+2\left(k_{6} \Delta \mathcal{D} \log n\right)
\end{aligned}
$$

rounds which is $O(\Delta D \log n)$.

Until this stage we proved that after expected $O(\Delta \mathcal{D} \log$ $n$ ) rounds the system reaches a forest configuration with a single leader and this leader is fixed forever i.e., the system reaches a leader configuration. To complete the proof and show that the system reaches a safe configuration we will show that the (single) root does not repeatedly extend its identity (due to wrong information on the existence of other trees). In the sequel we prove that such extension could happen at most once. We show that the root executes propagation of tid in the system and gets feedback after the propagation is terminated.

\subsubsection{Propagation of Information and Feedback}

In this stage of the proof we show that our protocol executes propagation of information (propagation of the new id of the root) and feedback (similarly to the protocol described in [29]) in $O(\Delta \mathcal{D})$ rounds (where $\mathcal{D}$ is the diameter of the communication graph). Note that the application of the protocol described in [29], to a shared memory system, requires $O(\Delta n)$ rounds. Unlike the protocol in [29] our protocol construct a breadth first tree (BFS tree) during the propagation of the new identity of the root and the feedback stage. The propagation and the feedback uses the tree to ensure that the time complexity is $O(\Delta \mathcal{D})$ rounds.

Let $E$ be an execution that starts with a leader configuration in which $P_{r}$ is the single root processor. By Lemma 9 no processor becomes a root and by Lemma 7 and Corollary 8 every configuration in $E$ define a forest, thus the single root processor is fixed throughout $E$. Informally, if $P_{r}$ extends $t i d_{r}$ during $E$ then following the first extension of the tid, the root floods the system with the new tid. Whenever a processor, $P_{i}$, assigns the new tid in $t i d_{i}$ for the first time in $E, P_{i}$ simultaneously assigns the color of the root in color $_{i}$ and assigns false in $a c k_{i}$ and in ot $_{i}$ (lines 5 to 13 of the code). We show that immediately before the root decides that the entire system is flooded with the new identifier (i.e., the condition in line 17 holds) the system reaches a leader configuration, $c$, that is called BFS configuration in which the following assertions hold:

- (bfs-1) FSG(c) is a BFS tree of the entire communication graph, with a single root processor $P_{r}$, and

- $\left(b f_{s}-2\right)$ for every nonroot processor $P_{l}$ with father $P_{f}$ it holds:

$$
\begin{aligned}
& \operatorname{tid}_{l}(c)=\operatorname{tid}[f](c)=\operatorname{tid}_{f}(c), \\
& \operatorname{dis}_{l}(c)=\operatorname{dis}_{l}[f](c)+1=\operatorname{dis}_{f}(c)+1, \\
& \operatorname{color}_{l}(c)=\text { color }_{l}[f](c)=\operatorname{color}_{f}(c), \operatorname{ack}_{l}(c)=\text { true, }
\end{aligned}
$$

and

$$
\text { ot }(c)=\text { false. }
$$

- (bfs-3) for every neighbor $P_{l}$ of $P_{r}$ it holds that $o_{r}[l]=$ false.

DEFINITION 7. Define a flooding subexecution,

$$
E^{\prime}=\left(c_{0}, \ldots, c_{b}\right),
$$


to be subexecution that starts with a leader configuration, $c_{0}$, that immediately follows an atomic step during which $P_{r}$ extends its tid (by executing line 20 of its program) and ends immediately before the next atomic step during which $P_{r}$ discovers that the flooding is over (the condition in line 17 holds).

Notice that by the definition of $E^{\prime}$, during $E^{\prime}, P_{r}$ does not extend its tid and does not change its color. Thus, for every configuration $c$ in $E^{\prime}, M T I D(c)=M T I D\left(c_{0}\right)$. Define $M T(c)$ to be the set of processors, $P_{i}$, in the configuration $c$ for which $t i d_{i}(c)=\operatorname{MTID}\left(c_{0}\right)$. For instance $M T\left(c_{0}\right)$ is the set of processors that includes $P_{r}$ solely. In the sequel we show that during $E^{\prime}$, the father-son relation in $M T(c)$ induces a subtree with root $P_{r}$ in $F S G(c)$ that includes all the processor with $t i d=M T I D(c)$.

LEMMA 17. If during flooding subexecution, $E^{\prime}=\left(c_{0}, \ldots, c_{b}\right), a$ nonroot processor $P_{j}$ changes its tid to $\operatorname{MTID}\left(c_{0}\right)$, then after this change and till the end of $E^{\prime}$ the following assertions hold:

1) The tid of any processor in the directed path from $P_{j}$ to $P_{r}$ in the FSG is $\operatorname{MTID}\left(c_{0}\right)$.

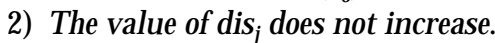

3) Every time $P_{j}$ changes its father in FSG, $P_{j}$ simultaneously decreases the value of dis $j_{j}$.

4) $\operatorname{color}_{j}=\operatorname{color}_{j}[f]=$ color $_{r}$, where $P_{f}$ is the father of $P_{j}$.

5) $o t_{j}=$ false.

6) In any configuration in which ack $=$ true it hold for every neighbor $P_{k}$ of $P_{j}$ that $t i d_{k}=t i d_{j}[k]=M T I D\left(c_{0}\right)$ and $d i s_{k} \leq$ $d i s_{j}[k] \leq d i s_{j}+1$.

7) Every time $P_{j}$ changes the value of ack ${ }_{j}$ to false, $P_{j}$ simultaneously decreases the value of dis . $_{j}$

PROOF.

1) By Lemma 4 during flooding subexecution $E^{\prime}$, after a processor $P_{j}$ that is not a root changes the value of $t i d_{j}$ to $\operatorname{MTID}\left(c_{0}\right)$ it holds that $t i d_{j}=\operatorname{MTID}\left(c_{0}\right)$. Hence by the $\left.{ }^{*}\right)$ relation of Lemma 6 , the tid of every processor in the directed tree from $P_{j}$ to the root of the tree is equal to $\operatorname{MTID}\left(c_{0}\right)$.

2) By Lemma 4 when $t i d_{j}$ is not changed $d i s_{j}$ may only be decreased, hence 2 .

3) The value in $t i d_{j}$ is not changed after the assignment of $M T I D\left(c_{0}\right)$ in $t i d_{j} . P_{j}$ changes its father (following the execution of lines 8 to 13 of the code) only when the value of $\left(t i d_{j}, d i s_{j}\right)$ grows, hence 3 .

4) We prove that during $E$ it holds for every nonroot processor in $M T, P_{j}$, with father $P_{f}$ that color $_{j}=\operatorname{color}_{j}[f]$ $=$ color $_{r}$. The proof is by induction on $i$, the index of the configuration in the execution. The induction base is by the fact that in $c_{0}$ the root processor is the only processor in $\operatorname{MT}\left(c_{0}\right)$. We assume that the induction assumption holds for $c_{i}$ and we prove that it holds for

$c_{i+1}$ where $c_{i} \stackrel{a_{i}+1}{\rightarrow} c_{i}+1$. Clearly, the induction assumption holds in $c_{i+1}$ when during $a_{i+1}$ no processor in MT writes in its color field and no processor joins MT. If during $a_{i+1}$ a processor in $\operatorname{MT}\left(c_{i}\right)$ writes in its color field then it must copy its father's color that is in $M T\left(c_{i}\right)$ and hence by the induction assumption it holds that $\operatorname{color}_{j}\left(c_{i+1}\right)=\operatorname{color}_{j}[f]\left(c_{i+1}\right)=\operatorname{color}_{r}\left(c_{i+1}\right)$. Whenever a processor joins MT the processor chooses one of the processors in $M T\left(c_{i}\right)$ to be its father and copies its color. Hence, also in that case the induction assumption hold in $c_{i+1}$.

5) Now we show that during $E$ ot $t_{j}=$ false for every nonroot processor in MT. When a processor joins MT the processors assigns ot: $=$ false. Assume for a moment that there is a processor $P_{j}$ that assigns ot $t_{j}:=$ true after joining $M T$. Let $P_{j}$ be the first processor in MT that assigns ot $t_{j}:=$ true. $P_{j}$ does not have sons in $M T$ with $o t=$ true, hence $P_{j}$ must discover a neighbor with different color from its own color (and its father color). The condition to check the neighbors' color (line 24) is that the color of the father of $P_{j}$ is different from the color of $P_{j}$, however by Assertion 4 above that condition does not hold.

6) After $P_{j}$ changes the value of $t i d_{j}$ to hold $\operatorname{MTID}\left(c_{0}\right) P_{j}$ assigns $a c k_{j}$ : $=$ true only when for every neighbor $P_{k}$ of $P_{j}$ it holds that $t i d_{j}[k]=\operatorname{MTID}\left(c_{0}\right)$ and $d i s_{j}[k] \leq d i s_{j}+1$ (line 14). Thus, all the neighbors of $P_{k}$ joined $M T$ and $P_{j}$ read the value of $\left(t i d_{k}, d i s_{k}\right)$ at least once after $P_{k}$ joined MT. Hence every further read of $P_{j}$ keeps the relation $t i d_{j}[k]=\operatorname{MTID}\left(c_{0}\right)$. Following the assignment ack $:=$ true $P_{j}$ changes the value of $d i s_{j}$ only if it simultaneously assigns $a c k_{j}:=$ false. Thus while $a c k_{j}=$ true the relation $d i s_{k} \leq d i s_{j}[k] \leq d i s_{j}+1$ that was true when $P_{j}$ assigns $a c k_{j}:=$ true is preserved.

7) A nonroot processor $P_{j}$ may assign $a c k_{j}$ : $=$ false only if it simultaneously increases the value of $\left(t i d_{j}, d i s_{j}\right)$ (line 13 of the code) or when color $_{j} \neq$ color $_{j}[f]$ (line 28). By Assertions 1 and 4 above color ${ }_{j}=\operatorname{color}_{j}[f]$ and $t i d_{j}=$ $\operatorname{MTID}\left(c_{0}\right)$ during $E^{\prime}$ and hence dis $s_{j}$ must decrease simultaneously with the assignment of $a c k_{j}:=$ false.

LEMMA 18. Let $E^{\prime}=\left(c_{0}, \ldots, c_{b}\right)$ be a flooding sub-execution, and $c^{\prime}$ a configuration in $E^{\prime}$, that immediately follows atomic step during which a processor $P_{j}$ with tid $_{j}=$ $\operatorname{MTID}\left(c_{0}\right)$ changes the value of ack $k_{j}$ to true. Then for every son, $P_{s}$ of $P_{j}$ in $F S G\left(c^{\prime}\right)$, ack $k_{s}\left(c^{\prime}\right)=$ true.

PROOF. Let $P_{s}$ be an arbitrary son of $P_{j}$ in $c^{\prime}$. Whenever $P_{j}$ joins $M T, P_{j}$ assigns $a c k_{j}:=$ false. After each time $P_{j}$ assigns $a c k_{j}:=$ false $P_{j}$ does not change $a c k_{j}$ to true before it reads and finds that $t i d_{s}=\operatorname{MTID}\left(c_{0}\right)$ and $d i s_{s} \leq d i s_{j}+1$.

We prove that $a c k_{s}=$ true in $c^{\prime}$. Let $c_{r}$ be a configuration that immediately follows the last read operation of $P_{j}$ from $P_{s}$ before $c^{\prime}$. In $c_{r} \operatorname{tid} d_{s}\left(c_{r}\right)=\operatorname{MTID}\left(c_{0}\right)$ and $\operatorname{dis}_{s}\left(c_{r}\right) \leq \operatorname{dis_{j}}\left(c_{r}\right)+1$. Now we show that in $c_{r} P_{s}$ is the son of $P_{j}$ and $a c k_{s}\left(c_{r}\right)=$ true.

First we prove that $P_{s}$ is a son of $P_{j}$ in $c_{r}$. Assume towards a contradiction that $P_{s}$ is not a son of $P_{j}$ in $c_{r}$ but is the son of $P_{j}$ in $c^{\prime}$. By Lemma 17 (3) $P_{s}$ changes its father after $c_{r}$ only when it simultaneously decreases the value of $d i s_{s}$. By the definition of $c_{r}$ it holds that $\operatorname{dis}_{s}\left(c_{r}\right) \leq \operatorname{dis}_{j}\left(c_{r}\right)+1$, thus if $P_{s}$ 
changes its father to be $P_{l}$ then $\operatorname{dis}_{l}\left(c^{\prime}\right)<\operatorname{dis}_{s}\left(c_{r}\right)-1 \leq$ $\operatorname{dis}_{j}\left(c_{r}\right)=\operatorname{dis}_{j}\left(c^{\prime}\right)$ and hence $P_{l} \neq P_{j}$ contradiction.

Now we show that $a c k_{s}\left(c^{\prime}\right)=$ true by the definition of $c_{r}$ and the fact that $P_{s}$ is the son of $P_{j}$ in $c_{r}$ it must hold that $a c k_{s}\left(c_{r}\right)=$ true. Assume towards contradiction that $P_{s}$ assigns $a c k_{s}:=$ false during the subexecution $E^{\prime \prime}$ that starts with $c_{r}$ and ends with $c^{\prime}$. By lemma 17 (7) during $E^{\prime \prime} P_{s}$ changes the value of ack to false only when it simultaneously decreases the value of $d i s_{s}$. Now we show that the value of $d i s_{s}$ does not decrease during $E^{\prime \prime}$. By the definition of $E^{\prime \prime} d i s_{j}$ is not changed during $E^{\prime \prime}$. Since it holds in $c_{r}$ that $\operatorname{dis}_{s}\left(c_{r}\right)=$ dis $\left(c_{r}\right)+1$ and since $P_{s}$ is the son of $P_{j}$ in $c^{\prime}$ it holds that $\operatorname{dis}_{s}\left(c^{\prime}\right)=\operatorname{dis}_{j}\left(c^{\prime}\right)+1$. Thus, the value of ack is not changed during $E^{\prime \prime}$.

LEMMA 19. In every flooding subexecution, $E^{\prime}=\left(c_{0}, \ldots, c_{b}\right), c_{b}$ is a BFS configuration.

PROOF. Define legal path in a configuration $c$ to be a directed path in $M T(c), \mathcal{P}(c)=\left(P_{1}, P_{2}, \ldots P_{l}\right)$ from the root $P_{1} \equiv$ $P_{r}$, to a leaf, $P_{l}$ that have the following property: there exist $0 \leq j \leq l$ such that for every $1 \leq \mathrm{k} \leq l$ it holds that if $k \leq j$ then $a c k_{k}(c)=$ false, and if $k>j$ then $a c k_{k}(c)=$ true.

First we prove by induction on $i$, the index of a configuration $c$ in a flooding subexecution $E^{\prime}$, the following claim:

InDUCTION Claim. Every path $\mathcal{P}(c)=\left(P_{1}, P_{2}, \ldots P_{l}\right)$ from the root, $P_{1} \equiv P_{r}$, to a leaf, $P_{l}$, of $M T(c)$ is a legal path.

INDUCTION BASE, $i=0 . c_{0}$ immediately follows an atomic step during which $P_{r}$ extends its tid to $\operatorname{MTID}\left(c_{0}\right)$ and in the same time assigns $a c k_{r}:=$ false. It is easy to see that in $c_{0}$, the induction assumption holds.

INDUCTION STEP. we assume that the induction assumption is true till $c$, the $i$ th configuration in $E^{\prime}$, and we prove that it is true in $c^{\prime}$ the $i+1$ th configuration of $E^{\prime}$. The only atomic steps that have influence on the induction assumption are atomic steps that includes a write operation that changes the values of $f$, tid, dis or ack. Changes in the values of $f$, tid, dis and ack of the processors in MT during $E^{\prime}=\left(c_{0}, \ldots, c_{b}\right)$ can occur by the execution of lines 12,13, 28, and 33 of the code. By the fact that $c_{0}$ is a forest configuration, line 12 is not executed during $E^{\prime}$. By Lemma 17 (4) the color of a processor in MT is equal to the color the processor read from its father and hence by the condition of line 24, line 28 is not executed either. Thus, the only atomic steps that changes the values of $f$, tid, dis, and ack in $M T$ are the atomic steps during which:

- Case 1: a new processor joins MT (executes line 13 of the code)

- Case 2: a processor in MT changes its father in the tree (executes line 13 of the code)

- Case 3: a processor in MT changes the value of ack to true (executes line 33 of the code)

We consider each of the above cases:
Case 1. a new processor, $P_{m}$, joins $M T$ as the son of a processor $P_{f}$ : since $t i d_{m}(c) \neq M T I D\left(c_{0}\right)$ then by lemma 17 (6) $a c k_{f}(c)=$ false (i.e., $P_{f}$ does not assigns $a c k_{f}:=$ true before $P_{m}$ assigns $\operatorname{MTID}\left(c_{0}\right)$ in $\left.t i d_{m}\right)$. Therefore, by the induction assumption it holds in $c$ for every processor, $P_{k}$ in the path $P_{1} \equiv P_{r}, \ldots, P_{l-1} \equiv P_{f}$ that $a c k_{k}(c)=$ false. Whenever $P_{m}$ joins $M T, P_{m}$ assigns $a c k_{m}$ : = false, hence the induction assumption holds for any path that includes $P_{m}$ in $M T\left(c^{\prime}\right)$. All the other paths in $M T\left(c^{\prime}\right)$ are identical to the paths in $M T(c)$, thus by the induction assumption they are legal paths in $c^{\prime}$ too.

Case 2. a processor $P_{m}$ in $M T$ changes its father in the tree to be $P_{f}$ : Let $\mathrm{P}$ be a path in $M T\left(c^{\prime}\right)$. If $\mathrm{P}$ is a path in $M T(c)$ too then by the induction assumption $\mathrm{P}$ is a legal path. Otherwise, if $\mathrm{P}$ is a prefix of a path in $M T(c)$ then since every prefix of a legal path is a legal path the path $\mathrm{P}$ is legal.

We still have to check a path $\mathcal{P}$ which is a concatenation of two parts of legal paths $\mathcal{P}=\mathcal{P}_{1}{ }^{\circ} \mathcal{P}_{2}$, when $\mathcal{P}_{1}$ is a prefix of a legal path in $M T(c)$ that is ended in $P_{f}$, and $\mathcal{P}_{2}$ is a suffix of a legal path in $M T(c)$, that begins in $P_{m}$. By Lemma 17 (3) it holds that such a concatenation occurs only when $\operatorname{dis}_{m}(c)>d i s_{f}(c)+1$. In such a case, by Lemma $17(6), a c k_{f}(c)=$ false, and by the induction assumption for every $P_{k} \in \mathcal{P}_{1}$ it holds that $a c k_{k}(c)=$ false. $\mathcal{P}_{2}$ is a suffix of legal path in $c$ that begins in $P_{m}$. Hence, in $c \mathcal{P}_{2}$ is a concatenation of a prefix with zero or more processors with ack $=$ false and a suffix of zero or more processors with ack = true. Hence in $c^{\prime}$ the concatenation $\mathcal{P}=\mathcal{P}_{1}{ }^{\circ} \mathcal{P}_{2}$ starts with two or more processors with ack $=$ false that are followed by zero or more processors with ack = true and hence $\mathcal{P}$ is a legal path. Thus, the induction claim is true in $c$ also in that case.

Case 3: a processor $P_{m}$ in MT changes the value of $a c k_{m}$ to true: By Lemma 18, it holds in $c^{\prime}$ for every son, $P_{s}$, of $P_{m}$ that $a c k_{s}\left(c^{\prime}\right)=$ true. Hence, every path that was a legal path in $c$ is also a legal path in $c^{\prime}$, and the induction assumption is true in $c^{\prime}$.

Now we complete the proof of the lemma: For every neighbor, $P_{k}$, of the root it holds in $c_{b}$ that $t i d_{k}\left(c_{b}\right)=$ $\operatorname{MTID}\left(c_{0}\right), \operatorname{dis}_{k}\left(c_{b}\right)=1$ and $a c k_{k}\left(c_{b}\right)=$ true. Since, all the paths in $M T\left(c_{b}\right)$ are legal paths and begins with a processor with ack $=$ true then for every processor $P_{i}$ that is not a root in $\operatorname{MT}\left(c_{b}\right)$, it holds that $a c k_{i}\left(c_{b}\right)=$ true. Thus, by Lemma 17 (6) it holds for every processor, $P_{i}$, in $M T\left(c_{b}\right)$ that all its neighbors are in $M T\left(c_{b}\right)$ too. By the definition of $c_{b}$ every neighbor of the root is in $M T\left(c_{b}\right)$, thus $M T\left(c_{b}\right)$ is a spanning tree of the entire communication graph. 
Now we prove that $F S G\left(c_{b}\right)$ is a BFS tree. We show that the value of $d i s_{m}$ of every processor, $P_{m}$, is equal to the distance $d_{m}$ of $P_{m}$ from $P_{r}$. Notice that by the fact that $c_{b}$ is a uniform tid configuration and by Lemma 6 it holds that $\operatorname{dis}_{m}\left(c_{b}\right) \geq d_{m}$. The proof is by induction on $d_{m}$. The induction base is due to the fact that $\operatorname{dis}_{r}\left(c_{b}\right)=0$. We assume that the induction assumption holds for every processor $P_{m}$ for which $d_{m} \leq$ $d$ and we prove that it is true for every processor $P_{l}$ such that $d_{l}=d+1$. By the induction assumption $P_{l}$ has a neighbor $P_{m}$ with $\operatorname{dis}_{m}\left(c_{b}\right)=d=d_{l}-1$. By Lemma 17 (6) $\operatorname{dis}_{l}\left(c_{b}\right) \leq d i s_{m}\left(c_{b}\right)+1$, it holds that $\operatorname{dis}_{l}\left(c_{b}\right)$ $\leq d+1$ and hence $d i s_{l}\left(c_{b}\right)=d+1$.

By the above $F S G\left(c_{b}\right)$ is a BFS tree, thus property $(b f s-1)$ of a BFS configuration holds. By the facts that in $c_{b}$ all the processors with tid $=\operatorname{MTID}\left(c_{0}\right)$ and $\operatorname{FSG}\left(c_{b}\right)$ is a $B F S$ tree that includes all the processors it holds in $c_{b}$ for every processor that is not the root, $P_{l}$, with father $P_{f}$, that:

$\operatorname{tidl}\left(c_{b}\right)=\operatorname{tid}[f]\left(c_{b}\right)=\operatorname{tid}\left(c_{f}\right)$, (due to Assertions 1 and 6 of Lemma 17), $d i s_{l}\left(c_{b}\right)=\operatorname{dis}[f]\left(c_{b}\right)+1=\operatorname{dis}_{f}\left(c_{b}\right)+1$, (due to Assertion 6 of Lemma 17 and the fact that for every processor $\left.P_{m} \operatorname{dis}_{m}\left(c_{b}\right)=d_{m}\right), \operatorname{color}_{l}\left(c_{b}\right)=\operatorname{color}_{l}[f]\left(c_{b}\right)=$ col$o r_{f}\left(c_{b}\right)$, (due to assertion (4) of Lemma 17), $a c k_{l}\left(c_{b}\right)=$ true (by the induction assumption above) and $o t_{l}\left(c_{b}\right)=$ false, (due to Assertion 5 of Lemma 17). Thus, property $(b f s-2)$ of a BFS configuration holds in $c_{b}$ too. Since after the root extends $t i d_{r}$ for the first time it holds for every neighbor with $t i d=t i d_{r}=\operatorname{MTID}\left(c_{0}\right)$ that $o t=$ false, and by the fact that $P_{r}$ reads from each of its neighbors $P_{m} t i d_{m}=\operatorname{MTID}\left(c_{0}\right)$ before $c_{b}$, property $(b f s-3)$ of a BFS configuration holds too.

THEOREM 20. During any execution $E$, that starts with a leader configuration, the root extends its tid at most once.

PROOF. Assume towards a contradiction that the root extends its tid more than once. By Lemma 19 after the first time that $P_{r}$ extends its tid and before the second time that $P_{r}$ extends its tid during $E$, a BFS configuration, $c_{b}$, is reached. In $c_{b}$ it holds for every neighbor $P_{l}$ of $P_{r}$ that ${ }_{0} t_{r}[l]=$ false. Thus, during the atomic step that follows $c_{b}, P_{r}$ chooses a new color (line 21 of the code) and does not extend its tid.

Now we trace a subexecution $E^{\prime}$ that starts in a BFS configuration, $c^{\prime}$, (in particular, $c_{b}$ ) and ends just before the next atomic step of the execution during which $P_{r}$ finds that the next coloring is finished (the condition in line 17 holds). We will show that during $E^{\prime}$ the $t i d$ of the root is not extended and $E^{\prime}$ terminates in a configuration $c_{b}^{\prime}$ that is a $B F S$ configuration. Hence the subexecution, that begins with $c_{b}^{\prime}$ and ends exactly before the following atomic step in which $P_{r}$ discovers that the coloring is finished, reaches a BFS configuration without extending the tid of the root. In such a way we can repeatedly use the same claims forever.

We prove that during $E^{\prime}$ the FSG is fixed and the global synchronization protocol is stabilized and executed in the right fashion on the fixed FSG. Notice that by the definition of $E^{\prime}$, during $E^{\prime}, P_{r}$ does not extend its tid and does not change its color. Thus for every configuration $c$ in $E^{\prime}$, the tid of a processor equals $M T I D\left(c^{\prime}\right)$. Moreover, since $c^{\prime}$ is a BFS configuration the value of dis of every processor is the distance of the processor from the root. Thus, it holds for every two neighbors $P_{i}$ and $P_{j}$ that $\left|d i s_{j}\left(c^{\prime}\right)-\operatorname{dis}_{i}\left(c^{\prime}\right)\right| \leq 1$. A processor changes its father only when the processor finds that there is a neighbor $P_{j}$ such that $t i d_{i}>t i d_{j}$, or when $t i d_{i}=t i d_{j}$ and $d i s_{i}<d i s_{j}-1$. Since the tid of all the processors is equal to $\operatorname{MTID}\left(c^{\prime}\right)$ and since the value of dis of every processor is the distance of the processor from the root no processor changes its father in the FSG during $E^{\prime}$. Thus, during $E^{\prime} F S G$ is a fixed BFS tree.

Since during $E^{\prime}$ the tree that is defined by $F S G\left(c^{\prime}\right)$ is not changed and since for every processor $P_{l}$ with father $P_{f}$ in $c^{\prime}$ it holds that

$$
\operatorname{color}_{l}\left(c^{\prime}\right)=\operatorname{color}_{l}[f]\left(c^{\prime}\right)=\operatorname{color}_{f}\left(c^{\prime}\right),
$$

the global synchronization protocol is already stabilized during $E^{\prime}$. Therefore whenever the root finds that all its sons acknowledge that they finished the coloring the system is in configuration $c_{b}^{\prime}$ in which for every nonroot processor $P_{l}$ with father $P_{f}$ in the system it holds that $a c k_{l}\left(c_{b}^{\prime}\right)=$ true and

$$
\operatorname{color}_{l}\left(c_{b}^{\prime}\right)=\operatorname{color}_{l}[f]\left(c_{b}^{\prime}\right)=\operatorname{color}_{f}\left(c_{b}^{\prime}\right) .
$$

As mentioned above, during $E^{\prime}$ no processor changes its $t i d$, dis and $f$, thus in order to prove that $c_{b}^{\prime}$ is indeed a BFS configuration it remains to show that for every nonroot processor $P_{l}$ it holds that ${ }_{0} t_{l}\left(c_{b}^{\prime}\right)=$ false.

By Lemma 2 during $E^{\prime}$ the color of every processor is changed exactly once. The color is changed from color $_{r}\left(c^{\prime}\right)$ to color $_{r}\left(c_{b}^{\prime}\right)$. In $c^{\prime}$ it holds for every processor that $o t\left(c^{\prime}\right)=$ false. We now show that no processor assigns true in ot during $E^{\prime}$. Assume towards a contradiction that there is a processor that assigns ot: $=$ true during $E^{\prime}$. Let $P_{l}$ be the first processor that assigns ot $t_{l}$ : $=$ true during $E^{\prime}$. Again by Lemma 2 during $E^{\prime} P_{l}$ executes that assignment only after it finds that its fathers' color is color $r\left(c_{b}^{\prime}\right)$ while color ${ }_{l}=\operatorname{color}_{r}\left(c^{\prime}\right)$ (line 24). Hence, if $P_{l}$ assigns $o t_{l}$ : $=$ true then during $E^{\prime} P_{l}$ reads from one of its neighbors a color that is 
different from $\operatorname{color}_{r}\left(c_{b}^{\prime}\right)$ and from $\operatorname{color}_{r}\left(c^{\prime}\right)$ (line 26). Contradiction, since during $E^{\prime}$ the color of a processor is either $\operatorname{color}_{r}\left(c_{b}^{\prime}\right)$ or $\operatorname{color}_{r}\left(c_{b}^{\prime}\right)$. Hence $P_{l}$ does not as-

sign $o t_{l}:=$ true and hence the last configuration of $E^{\prime}$ is a BFS configuration.

Note that by Lemmas 10 , and 11 , and Corollary 12 it holds that $O(\Delta \mathcal{D})$ rounds after the root extends its tid the flooding of the new tid is ended. Thus the time complexity of the propagation of information and feedback protocol is $O(\Delta \mathcal{D})$ rounds.

\section{RANKING}

In this section we present a self-stabilizing protocol which ranks the systems' processors. First we present a selfstabilizing ranking protocol that works on systems whose communication graph is an in-tree. This protocol assigns each processor a rank which equals its DFS number. The time complexity of this protocol is $O(\Delta D)$ rounds, and its space complexity is $O(\Delta \log n)$. Then we use the technique of fair protocol combination of [15], and achieve a uniform self-stabilizing ranking protocol for general graphs by composition of this protocol with the protocol presented in the previous section. The time and space complexities of the combined protocol are the sum of the complexities of the two protocols.

The ranking protocol, for a tree system, is also a composition of two other protocols that are composed by a fair protocol composition. In one of those protocols each processor $P_{j}$ computes the number of processors, $n_{j}$, in the subtree rooted at $P_{j}$, this protocol is called counting protocol. The second protocol is called the naming protocol. The naming protocol provides a distinct name for every processor. Each of those protocols stabilizes within $O(\Delta D)$ rounds and the space complexity of a processor is $O(\Delta \log n)$.

\subsection{Counting Protocol}

Every processor $P$ has a register in which $P$ writes to its father in the tree. A leaf processor writes in every atomic step the value 1 to its father. A non leaf processor repeatedly reads its sons' registers, sums their values, adds one to this sum, and writes the result to its father. Define the height of a processor $Q$ in a tree to be the maximal number of processors in a path that starts in a leaf and ends in $Q$. The correctness and complexity proof of the counting protocol is by induction on the height of the processors. The induction assumption is that following $O(\Delta h)$ rounds the register of a processor, $Q$, that is in height less than or equal to $h$, holds the number of processors in the subtree rooted at $Q$. The induction base is by the fact that every processor that is in height 0 is a leaf. The induction step is derived from the induction assumption for processors in height $h$ and the way processors in height $h+1$ calculate the number of processors in their subtree.

\subsection{Naming Protocol}

Every processor, $P$, orders its sons, $\left(P_{1}, P_{2}, \ldots, P_{i}\right)$ and uses a register to write to each of his sons. Let $n_{1}, n_{2}, \ldots, n_{i}$ be the number assigned by the counting protocol for $P_{1}, P_{2}, \ldots, P_{i}$, respectively, and read by $P$. The root chooses the identity 1 and repeatedly writes it to its sons. The root writes the value $2+n_{1}+n_{2}+\ldots+n_{j-1}$ to its $j$ son. A nonroot processor $Q$ repeatedly reads the number in its father register and choose its identity to be that value, say the value $x$. $Q$ repeatedly writes to each of its sons. $Q$ writes the value $x+1$ $+n_{1}+n_{2}+\ldots+n_{j-1}$ to its $j$ son. Define the depth of a processor $Q$ in an in-tree to be the number of processors in the directed path that starts in $Q$ and ends in the root. The correctness and complexity proof of the naming protocol is by induction on the depth of the processors. The induction assumption is that following $O(\Delta d)$ rounds every processor, $Q$, that is in depth less than or equal to $d$, chooses an identity that is equal to its DFS index in the tree. The induction base is by the fact that the root processor chooses the identity 1 . The induction step is derived from the induction assumption for processors in depth $d$ and the way processors in height $d$ and height $d+1$ communicate.

\section{Conclusions}

We presented a uniform self-stabilizing leader election protocol. In a uniform system processors do not have unique identifiers. The protocol uses randomization in order to break symmetry. Our protocol is the only protocol which solves the problem without any prior knowledge on the communication graph. Self-stabilizing local and global synchronization protocols are used as building blocks for the leader-election protocol. Those protocols can be used to implement many distributed tasks as synchronizers and reset protocols. Part of the self-stabilizing leader election is a new propagation of information with feedback protocol that terminates within $\Omega(\Delta \mathcal{D})$ rounds.

The self-stabilizing leader election protocol and ranking protocols can be combined, by the fair protocol combination method introduced in [15], with other self-stabilizing protocols that assume either a unique leader or unique identifiers. Thus, the vocabulary of self-stabilizing protocols for uniform distributed systems is enriched.

Throughout the presentation of the leader election protocol in Section 3 it is assumed that a processor can toss a single coin in a single atomic step. Under this restriction it is proven that a leader is elected in $O(\Delta \mathcal{D} \log n)$ rounds. It is easy to verify that when $O(\log n)$ coin tosses are executed in a single atomic step then the protocol stabilizes in $\Omega(\Delta \mathcal{D})$ rounds. Note that $\Omega(\Delta \mathcal{D})$ rounds are required to convey information from one side of the system to the other and thus to elect a leader.

Note that following the stabilization phase of our leader election protocol the protocol repeatedly colors a spanning tree of the system communication graph. Hence, the protocol can be used as a self-stabilizing synchronizer ( $\beta$ synchronizer in the terms of [9]) that converts self-stabilizing synchronous protocols to work in asynchronous system. One of the anonymous referees point out the relation to gossiping algorithms e.g. [13], [19], where processors communicate information among themselves. Our protocol can sport self-stabilizing gossiping easily by repeatedly collecting the information to the elected leader through the spanning tree and then broadcasting it along the tree. Another possibility is to use the virtual ring defined by a DFS 
tour on the spanning tree to forward information to the destination and to use hop-counter to eliminate corrupted information from the system.

\section{APPENDIX-NOTATIONS AND TERMS}

\begin{tabular}{|c|c|}
\hline$\Delta$ & the maximal degree of a node in the graph. \\
\hline $\mathcal{D}$ & the diameter of the graph. \\
\hline$n$ & the number of processors in the graph. \\
\hline loop iteration & see definition 1 and 2 . \\
\hline read iteration & see definition in 2 . \\
\hline$F S G(c)$ & $\begin{array}{l}\text { the father-son relation graph of the configura- } \\
\text { tion } c . F S G \text { consist of a directed edge from } \\
\text { each non-root processor to its father. }\end{array}$ \\
\hline tid & $\begin{array}{l}\text { the tree identifier to which a processor be- } \\
\text { longs. }\end{array}$ \\
\hline dis & the distance of a processor from its root. \\
\hline color & the variable used to repeatedly color a tree. \\
\hline ack & $\begin{array}{l}\text { a variable used to acknowledge the termina- } \\
\text { tion of the coloring procedure in the subtree } \\
\text { the processor. }\end{array}$ \\
\hline ot & $\begin{array}{l}\text { Boolean indication on the existence of other } \\
\text { trees. }\end{array}$ \\
\hline $\begin{array}{l}\text { leader configu- } \\
\text { ration }\end{array}$ & $\begin{array}{l}\text { a configuration with exactly one leader such } \\
\text { that in any execution that starts with this con- } \\
\text { figuration the leader is fixed. }\end{array}$ \\
\hline $\begin{array}{l}\text { forest configura- } \\
\text { tion }\end{array}$ & see definition 3. \\
\hline$R(c)$ & the set of root processors in configuration $c$. \\
\hline MTID & see definition 5 . \\
\hline $\begin{array}{l}\text { uniform tid con- } \\
\text { figuration }\end{array}$ & see definition 5 . \\
\hline $\begin{array}{l}\text { utff-execution } \\
\text { flooding } \\
\text { subexecution }\end{array}$ & $\begin{array}{l}\text { uniform tid fixed forest execution, } \\
\text { see definition } 7 \text {. }\end{array}$ \\
\hline
\end{tabular}

\section{ACKNOWLEDGMENT}

We would like to thank the anonymous referees for their constructive remarks that helped to improve this paper. S. Dolov's work was supported in part by TAMU Engineering Excellence funds and the National Science Foundation's Presidential Young Investigator Award CCR-9158478. Part of his work was done while Dr. Dolov was at Technion and Texas A\&M University.

\section{REFERENCES}

[1] K. Abrahamson, A. Adler, L. Higham, and D. Kirkpatrick, "Probabilistic Solitude Verification on a Ring," Proc. Fifth Ann. ACM Symp. Principles of Distributed Computing, Quebec City, pp. 161-173, Aug. 1986.

[2] H. Attiya, M. Snir, and M. Warmuth, "Computing on an Anonymous Ring," J. ACM, vol. 35, no. 4, pp. 845-875, Oct. 1988.

[3] K. Abrahamson, "On Achieving Consensus Using a Shared Memory," Proc. Seventh Ann. ACM Symp. Principles of Distributed Computing, pp. 291-302, Toronto, Canada, Aug. 1988.

[4] E. Angnostou and R. El-Yaniv, "More on the Power of Random Walks: Uniform Self-Stabilizing Algorithms," Lecture Notes in Computer Science 579: Distributed Algorithms, Proc. Fifth Int'l Workshop Distributed Algorithms, S. Toueg, P.G. Spirakis, and L. Kirousis, eds, Delphi, Greece, pp. 31-51, Oct. 1991. Springer-Verlag, 1992.

[5] A. Arora and M. Gouda, "Distributed Reset," Lecture Notes Computer Science 472: Foundations of Software Technology and Theoretical Computer Science, Proc. 10th Conf. Foundations of Software Technology and Theoretical Computer Science, K.V. Nori and C.E. Veni Madhavan, eds., pp. 316-331, Dec. 1990. Springer-Verlag, 1990.
[6] Y. Afek, S. Kutten, and M. Yung, "Memory-Efficient SelfStabilization on General Networks," Lecture Notes in Computer Science 486: Distributed Algorithms, Proc. Fourth Int'l Workshop Distributed Algorithms, J. Van Leeuwen and N. Santoro, eds, pp. 15-28. Springer-Verlag, 1991.

[7] Y. Afek, S. Kutten and M. Yung, "Local Detection for Global SelfStabilization," preprint.

[8] B. Awerbuch and G. Varghese, "Distributed Program Checking a Paradigm for Building Self-Stabilizing Distributed Protocols," Proc. 31st Ann. IEEE Symp. Foundations of Computer Science, pp. 268-277, 1991.

[9] B. Awerbuch, "Complexity of Network Synchronization," J. ACM, vol. 32, no. 4, pp. 804-823, 1985.

[10] G.M. Brown, M.G. Gouda, and C.L. Wu, "A Self-Stabilizing Token System," Proc. 20th Ann. Hawaii Int'l Conf. System Sciences, pp. 218223, 1987.

[11] J.E. Burns and J. Pachl, "Uniform Self-Stabilizing Rings," ACM Trans. Programming Languages and Systems, vol. 11, no. 2, pp. 330344, Apr. 1989.

[12] J.E. Burns, "Self-Stabilizing Rings Without Demons," Technical Report GIT-ICS-8736, Georgia Inst. of Technology.

[13] B.S. Chlebus, K. Diks, and A. Pelc, "Fast Gossiping With Short Unreliable Messages," Discrete Applied Math., special issue on gossiping, vol. 53, pp. 15-24, 1994.

[14] E.W. Dijkstra, "Self-Stabilizing Systems in Spite of Distributed Control," Comm. ACM vol. 17, no. 11, pp. 643-644, 1974.

[15] S. Dolev, A. Israeli, and S. Moran, "Self Stabilization of Dynamic Systems," Proc. Ninth Ann. ACM Symp. Principles of Distributed Computing, Quebec City, pp. 103-118, Aug. 1990. Distributed Computting vol. 7, pp. 3-16, 1993.

[16] S. Dolev, A. Israeli, and S. Moran, "Uniform Dynamic SelfStabilizing Leader Election," in Lecture Notes in Computer Science 579: Distributed Algorithms, Proc. Fifth Int'l Workshop on Distributed Algorithms, Delphi, Greece, S. Toueg, P.G. Spirakis and L. Kirousis, eds., pp. 163-180, Oct. 1991. Springer-Verlag, 1992.

[17] S. Dolev, A. Israeli, and S. Moran, "Analyzing Expected Time by Scheduler-Luck Games," IEEE Trans. Software Eng., vol. 21, no. 5, pp. 429-439, May 1995.

[18] R. G. Gallager, "Finding a Leader in Networks With $O(E)+O(N$ $\log N)$ Messages," Internal Memo., M.I.T., Cambridge, Mass., 1978.

[19] S.M. Hedetniemi, S.T. Hedetniemi, and A.L. Liestman, "A Survey of Gossiping and Broadcasting in Comm. Networks," Networks, vol. 18, pp. 319-349, 1988.

[20] P. Humblet, "Selecting a Leader in a Clique in $O(N \log n)$ Messages. Internal Memo., Laboratory for Information and Decision Systems, M.I.T., Cambridge, Mass., 1984.

[21] A. Israeli and M. Jalfon, "Token Management Schemes and Random Walks Yield Self Stabilizing Mutual Exclusion," Proc. Ninth Ann. ACM Symp. Principles of Distributed Computation, Quebec City, pp. 119-132, Aug. 1990.

[22] A. Israeli and M. Jalfon, "Self Stabilizing Ring Orientation," Lecture Notes in Computer Science 486: Distributed Algorithms (Proc. Fourth Int'l Workshop on Distributed Algorithms, Bari, Italy, J. Van Leeuwen and N. Santoro, eds, pp.1-14, Sept. 1990. SpringerVerlag, 1991.

[23] A. Itai and M. Rodeh, "Probabilistic Methods for Breaking Symmetry in Distributed Networks," Information and Computation, vol. 88 , no. 1 , pp. $60-87,1990$.

[24] E. Korach, S. Kutten, and S. Moran, "A Modular Technique for the Design of Efficient Distributed Leader Finding Algorithms," ACM Trans. Programming Languages and Systems, vol.12, no. 1, pp. 84-101, 1990.

[25] E. Korach, S. Moran, and S. Zaks, "Tight Lower and Upper Bounds for Some Distributed Algorithms for Complete Network of Processors," Proc. Third Ann. ACM Symp. Principles of Distributed Computing, pp. 199-207, 1984.

[26] S. Katz and K.J. Perry, "Self-Stabilizing Extensions for MessagePassing Systems," Proc. Ninth Ann. ACM Symp. Principles of Distributed Computing, Quebec City, pp. 91-101, Aug. 1990.

[27] L. Lamport, "The Mutual Exclusion Problem: Part II-Statement and Solutions," J. ACM, vol. 33, no. 2, pp. 327-348, 1986.

[28] Y. Matias and Y. Afek, "Simple and Efficient Election Algorithms for Anonymous Networks," manuscript, 1989.

[29] A. Segall, "Distributed Networks Protocols," IEEE Trans. Comm., vol. 29, no. 1, pp. 23-35, Jan. 1983. 
[30] B. Schieber and M. Snir, "Calling Names on Nameless Networks," Proc. Eighth Ann. ACM Symp. Principles of Distributed Computing, Edmonton, pp. 319-328, Aug. 1989.

[31] M. Tchuente, "Sur l'auto-stabilisation dans un réseau d'ordinateurs," RAIRO Inf. Theor., vol. 15, pp. 47-66, 1981.

Shlomi Dolev received his BSc in engineering and BA in computer science in 1984 and 1985, respectively, and his MSc and PhD in computer science in 1990 and 1992, respectively, from the Technion Israel Institute of Technology. From 1992 to 1995 he was at Texas A\&M University as a visiting research specialist. He visited Carleton University in 1994. In 1995 he joined the Department of Mathematics and Computer Science at Ben-Gurion University. His current research interest include the theoretical aspects of distributed computing and communication networks.
Amos Israeli received his BSc in mathematics and physics from $\mathrm{He}-$ brew University in 1976, and his MSc and DSc in computer science in 1980 and 1985, respectively. He was a postdoctoral fellow at the Aiken Computation Laboratory at Harvard. His research interests are in parallel and distributed computing and robotics. He has worked on the design and analysis of wait-free and self-stabilizing distributed protocols.

Shlomo Moran received his BSc and $\mathrm{PhD}$ degrees in Mathematics from the Technion in 1975 and 1979, respectively. In 1979-1981 he was at the University of Minnesota as a visiting research specialist. In 1981 he joined the Computer Science Department at the Technion, where he is now a full professor. In 1985-1986 he visited at IBM T.J. Watson Research Center. In 1992-1993 he visited at AT\&T Bell Laboratories and in Centrum voor Wiskunde en Informatica, Amsterdam. His research interests include distributed computing, combinatorics and graph theory, and complexity theory. 